\title{
Geological monitoring networks for risk management close to large rock cliffs: the case history of Gallivaggio and Cataeggio in the italian Alps
}

\author{
Luca Dei Cas ${ }^{1}$, Maria Luisa Pastore ${ }^{2}$, Andrea Pavan ${ }^{1}$, and Nicola Petrella ${ }^{1}$ \\ ${ }^{1}$ Arpa Lombardia U. O. Centro di Monitoraggio Geologico, Via del Gesù 17, 23032 Sondrio, Italy \\ ${ }^{2}$ Arpa Lombardia Settore Rischi Naturali, Via Rosellini 11, 22100 Milano, Italy \\ Correspondence: Luca Dei Cas (1.deicas@arpalombardia.it)
}

Received: 1 April 2020 - Revised: 24 February 2021 - Accepted: 24 February 2021 - Published: 1 April 2021

\begin{abstract}
In areas located near large rock cliffs, risk reduction by early warning monitoring systems highligts potentiality but also critical issues and limits. The paper examines two rock slope failures that occurred in a short time from each other near inhabited areas in the Italian Alps. The viscous behavior of the rock mass was reconstructed through data processing from ground-based Synthetic Aperture Radar Interferometry (InSAR), and elaboration of acceleration and speed curves. Landslides types and underlying complexity associated with rock detachment mechanisms suggest the identification of precautionary alarm thresholds for collapse forecasting. The analysis of financial outlay, both for mitigation works and for monitoring activities, highlight the adequacy and the opportunity to combine passive systems, like embankments or rockfall drapery meshes, with a reliable monitoring network for early warning.
\end{abstract}

\section{Introduzione}

Nella parte di mondo tecnologicamente più sviluppata la richiesta di sicurezza rispetto ai fenomeni naturali è sempre più elevata (Macciotta et al., 2016). Mentre spesso gli abitanti rifiutano la logica di un utilizzo territoriale equilibrato e compatibile alla pericolosità dei propri territori, paradossalmente da parte degli stessi aumenta la richiesta di sicurezza. In altre parole, il cittadino è diventato consumatore di sicurezza come di un servizio che gli è dovuto, a prescindere dai suoi comportamenti e dalle conseguenze delle scelte da lui adottate (Gabrielli, 2013).

In aggiunta a ciò, bisogna evidenziare che le problematiche socioeconomiche legate a frane e dissesti mettono l'Italia al primo posto europeo (Frattini e Crosta, 2006) e più in generale i danni da frana causano nel mondo, ogni anno, centinaia di milioni di dollari di danni (Klose et al., 2014).

Anche per tale ragione la mitigazione e gestione dei rischi naturali costituisce un problema per le società avanzate (Leroi et al., 2005) e ha comportato forti sviluppi nelle tecniche legate al monitoraggio geologico. A tal proposito in Regione Lombardia (Italia), a partire dall'ultimo decennio del secolo scorso, si è sviluppato uno strutturato Centro di Monitoraggio Geologico che a oggi tiene sotto stretta sorveglianza oltre quaranta importanti frane, distribuite sul territorio alpino, prealpino e appenninico del territorio regionale, mediante altrettante reti di monitoraggio.

Le reti di monitoraggio geologico sono opere di difesa non strutturale che necessitano di progettazione (Dei Cas, 2014) e che, nel caso dell'early warning, sono finalizzate alla riduzione della probabilità che persone o strutture mobili presenti possano venir convolte in un evento di frana (Sättele et al., 2015). La sola realizzazione di un sistema di monitoraggio non è però sufficiente a dispiegare questo benefico effetto di diminuzione del rischio da frana $(R)$ che, secondo la classica formula, è ottenuto dal prodotto di pericolosità $(P)$, vulnerabilità dell'area interessata $(V)$ e valore $(W)$ degli elementi a rischio (Varnes, 1984). Per ridurre il rischio, dopo aver predisposto una rete di monitoraggio, si dovrà procedere alla stesura di uno studio specifico che definirà puntuali scenari di evento e soglie di allertamento da applicarsi alla strumentazione installata; al superamento di predetti valori soglia sarà necessario aver previsto una apposita pianificazione, aggiornata e nota a tutti 
i soggetti coinvolti, che permetterà di dispiegare rapidamente procedure di protezione civile (evacuazioni, interruzioni della viabilità, rimozione strutture/opere di valore ecc....) (Intrieri et al., 2013). La mancanza, anche solo di uno di questi passaggi, può diminuire fortemente l'efficacia, nell'accezione più ampia del termine, che il monitoraggio geologico ha sulla riduzione del rischio di frana.

In letteratura (Sättele et al., 2015) l'efficacia previsionale $(E)$ di un sistema di monitoraggio è funzione esclusivamente della capacità di rilevare un evento (PDO Probability of detection) rispetto al numero di falsi allarmi (PFA Probability of False Alarm) generati. Non si può però non tenere in conto che senza una adeguata gestione, intesa come insieme di azioni che dallo studio di modellazione del dissesto, passando per la predisposizione/aggiornamento del piano di protezione civile, giungano alla sottrazione di persone o strutture mobili dall'area potenzialmente interessata dallo scenario, l'efficacia della rete di monitoraggio venga fortemente depotenziata.

La gestione di una rete di monitoraggio, finalizzata all'allertamento, presenta gradi di difficoltà differenti in funzione della conoscenza del fenomeno, ma anche della tipologia del fenomeno stesso.

Fra i dissesti, oggetto di monitoraggio del Centro di Monitoraggio Geologico della Lombardia, ve ne sono alcuni che morfologicamente si presentano come pareti rocciose subverticali; dette pareti si mostrano come aree dalle quali potenzialmente possono generarsi distacchi sia di esigui quantitativi di roccia (blocchi singoli o di decine di metri cubi) sia di ammassi rocciosi di centinaia o migliaia di metri cubi.

È pertanto immediatamente comprensibile come tali tipologie di pareti rocciose rappresentino, per il monitoraggio geologico con finalità di early warning, una delle sfide più complicate e impegnative. Se è sostanzialmente nota l'impossibilità o l'estrema difficoltà di riuscire a gestire un sistema d'allarmistica che permetta di prevedere il momento del distacco e del crollo di singoli blocchi, sia per la dimensione ridotta dell'area da monitorare sia per l'estremamente ridotto tempo di sviluppo e accadimento, alcuni recenti esempi di monitoraggio incoraggiano a gestire un sistema di allarmistica per franamenti, con processi di deformazione e rottura di tipo viscoso, che coinvolgano migliaia di metri cubi di roccia.

Ovviamente tali reti di monitoraggio con funzioni di allarmistica devono misurarsi sia con le incertezze legate alla geometria, alla struttura e alla meccanica dei versanti (la persistenza delle discontinuità, le caratteristiche duttili o fragili degli ammassi, la forma delle superfici di scivolamento ecc.) sia con le difficoltà insite nella realizzazione di modelli previsionali finalizzati ad anticipare i meccanismi che regolano i distacchi in roccia.

Proprio tali incertezze si riflettono, relativamente alla gestione delle reti di early warning, nella definizione delle soglie. Queste non dovranno essere definite con

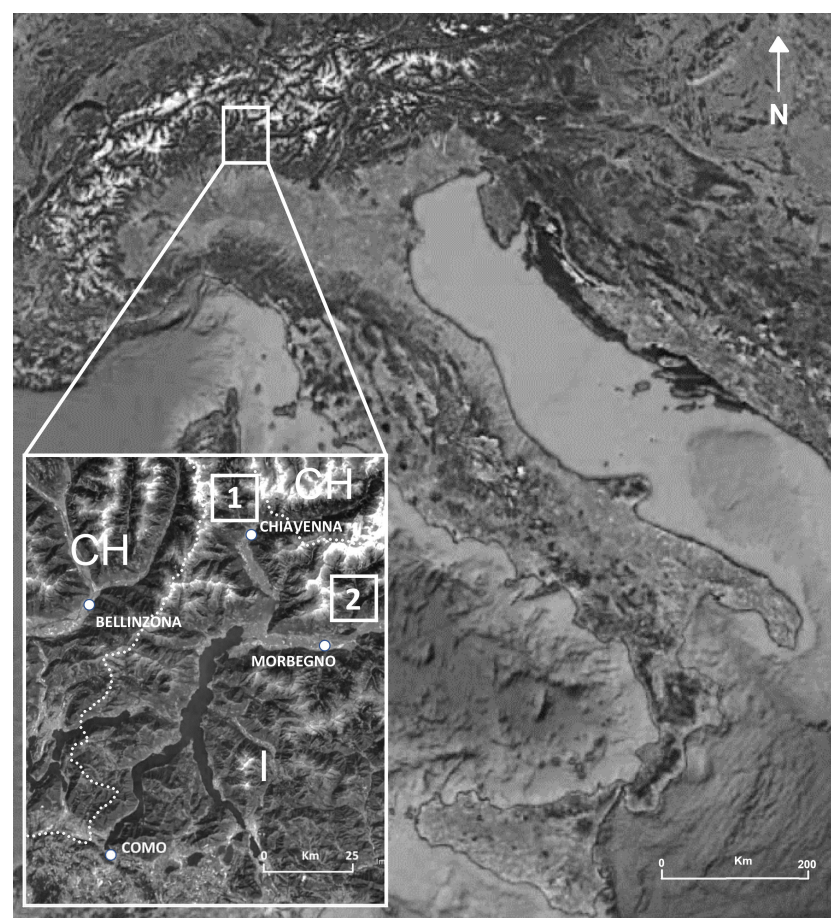

Figure 1. Inquadramento geografico delle aree di studio (1 Gallivaggio, 2 Cataeggio). (Mappa ISPRA - Geoportale Nazionale - "Italian Open Data License".)

valori troppo conservativi, ciò produrrebbe numerosi allarmi con importanti e poco giustificabili perdite economiche, ma d'altro canto andranno evitati valori troppo alti che metterebbero in pericolo le vite umane sia per eventuali mancati allarmi sia per l'emissione di preavvisi troppo prossimi al momento dell'evento.

Tenuto conto delle limitazioni e problematiche sopra esposte questo articolo prenderà in considerazione due eventi di scivolamento traslazionale in roccia (Varnes, 1978), evoluti in crolli stante la morfologia subverticale del versante e avvenuti a pochi mesi di distanza su due pareti rocciose delle Alpi lombarde.

I dati acquisiti dal monitoraggio in tempo reale, sia precedentemente sia durante il fenomeno di franamento, potranno rappresentare preziose informazioni quantitative per accrescere le conoscenze su fenomeni per i quali non vi è una chiara e completa conoscenza dei meccanismi (Eberhardt, 2008; Federico et al., 2012) che determinano il passaggio da stabilità a instabilità.

Nel presente testo verranno pertanto descritti e discussi due casi di studio (Fig. 1) relativi alla parete in roccia sovrastante il Santuario della Madonna di Gallivaggio, in particolare il franamento avvenuto il 29 maggio 2018, e a quella di Cataeggio, con attenzione puntata allo scivolamento avvenuto il 4 febbraio 2019 . 


\section{Metodologia}

Per il monitoraggio delle due pareti sono stati utilizzati radar da terra ad apertura sintetica del medesimo modello (GBInSAR LiSALab). L'utilizzo dell'interferometria con radar da terra mostra grandi vantaggi nello studio delle frane quali, fra gli altri, l'ampio areale di investigazione, la sicurezza degli operatori, la possibilità di seguire movimenti giornalieri da sub millimetrici a plurimetrici, la velocità d'installazione e il funzionamento con qualsiasi condizione di luce. Vanno però anche considerate le principali limitazioni determinate dalla necessità di avere una vista con distanza e angolazione favorevole, l'impossibilità di rilevare movimenti impulsivi e di acquisire dati su versanti coperti da vegetazione.

I radar, ubicati in posizione frontale rispetto alle pareti da monitorare, sono stati collocati all'interno di specifiche strutture con finestrature, protette da apposito radome, per permettere la corretta osservazione dall'area di indagine. Detti radar vengono alimentati elettricamente mediante rete fissa o con un sistema di continuità in caso di interruzione momentanea dell'alimentazione.

I dati così acquisiti vengono processati in loco, quindi pubblicati su apposita pagina web per l'analisi degli stessi e inviati in automatico a database remoti per l'integrazione negli specifici software di allertamento.

Il sistema utilizzato è costituito da uno scatterometro a microonde a onda continua, che tramite apposita antenna emette il segnale e ne riceve la retrodiffusione dopo che lo stesso ha colpito la parete.

Il sistema di antenne si muove lungo un binario fissato a terra per realizzare l'apertura sintetica. Il funzionamento in continuo prevede scansioni, per entrambe le pareti, ogni $2^{\prime} 30^{\prime \prime}$ circa. In assenza di movimenti o con movimenti estremamente lenti, l'analisi viene effettuata su lunghi intervalli di tempo così da poter avere una maggior precisione del dato, derivato da segnali affetti da inevitabili disturbi ambientali, con caratteristiche del decimo di millimetro.

La risoluzione spaziale delle immagini SAR dipende dai parametri di misura scelti, dalle caratteristiche dell'installazione e dalla distanza del sensore rispetto alla scena osservata. A Gallivaggio la risoluzione è pari a circa $0.75 \mathrm{~m}$ in range mentre quella in azimut varia tra $0.44 \mathrm{e}$ $2.17 \mathrm{~m}$. A Cataeggio la risoluzione è pari a circa $1.5 \mathrm{~m}$ in range mentre quella in azimut varia tra 0.9 e $2.6 \mathrm{~m}$.

I dati acquisiti con l'interferometria radar presentano la limitazione di essere immediatamente utilizzabili solo qualora, nell'intervallo di tempo dell'interferogramma (come minimo il tempo che intercorre fra una immagine radar e la successiva), sia avvenuto un movimento minore uguale di 0.25 della lunghezza dell'onda che, generata dal radar, è stata riflessa dal medesimo bersaglio. Poiché la lunghezza dell'onda utilizzata dai radar ad apertura sintetica è pari a $17.44 \mathrm{~mm}$, lo spostamento massimo rilevabile dagli interferogrammi è pari a $4.4 \mathrm{~mm}$ ogni $2^{\prime} 30^{\prime \prime}$. Siccome i dissesti analizzati, specialmente nelle ultime decine di minuti prima del crollo, hanno assunto velocità anche pari o superiori al centimetro ogni $2^{\prime} 30^{\prime \prime}$, è stato necessario sottoporre i dati acquisiti al processo dello srotolamento di fase (phase unwrapping) prima di poterli analizzare. Questo processo prevede l'analisi di ogni singolo interferogramma al fine di poter attribuire il corretto valore di spostamento ad aree o punti rilevati dal radar.

Per poter analizzare al meglio i dati acquisiti con i radar da terra ad apertura sintetica, è fondamentale disporre di un modello digitale del terreno aggiornato e con adeguata risoluzione spaziale. Nei casi oggetto di studio la restituzione tridimensionale dei versanti è stata eseguita mediante tecniche aerofotogrammetriche. Le immagini sono state riprese utilizzando droni DJI Phantom 4 RTK e Phantom 4 Pro in dotazione all'Agenzia Regionale per la Protezione dell'Ambiente (di seguito ARPA). Per ottenere la precisione topografica in fase di ripresa sono stati posizionati a terra dei punti di controllo, le cui coordinate sono state determinate con strumentazione GPS. Il modello digitale dei versanti e le relative Mesh sono stati realizzati con il software Pix $4 D$ Mapper, mentre il calcolo dei volumi e la definizione delle famiglie di piani di discontinuità degli ammassi rocciosi sono state eseguite mediante il software CloudCompare, dedicato all' analisi delle nuvole di punti create con i software per fotogrammetria. Le giaciture dei piani di discontinuità sono infine state elaborate con il software per stereogrammi Orient.

\section{Inquadramento geografico e geologico}

L'area del Santuario della Madonna di Gallivaggio si trova in Comune di San Giacomo Filippo, nella parte più occidentale del territorio della Provincia di Sondrio.

La parete subverticale che domina il Santuario (Fig. 2), strutturalmente appartenente alla Falda Tambò, delimita il fianco idrografico sinistro della bassa Valle Spluga. Il versante, con sviluppo verticale di oltre $500 \mathrm{~m}$ e inclinazione compresa fra 65 e $70^{\circ}$, è interamente costituito da un ortogneiss occhiadino conosciuto come Metagranito del Truzzo. Al piede della parete di Gallivaggio sono presenti accumuli sia sotto forma di coni e falde di detrito sia sotto forma di grossi blocchi e macereti, a testimonianza della intensa attività di crolli provenienti dalla parete. Gli ammassi rocciosi presentano sistemi di discontinuità dotati di una certa variabilità giaciturale in relazione ai disturbi strutturali presenti nell'area (Ferrari et al., 2011).

Mediante le attività con drone sono state individuate le fratture principali, rilevate nella porzione superiore della parete, che possono essere raggruppate nelle famiglie $\mathrm{K} 1 \mathrm{im}-$ mergente a SW (giacitura media $220 / 66^{\circ}$ ), $\mathrm{K} 2$ immergente a W $\left(279 / 68^{\circ}\right)$, K3 immergente a $\mathrm{N}\left(008 / 28^{\circ}\right)$ e $\mathrm{K} 4$ a SE $\left(118 / 77^{\circ}\right)$. Il piano, tutt'ora ben visibile, su cui è traslato 


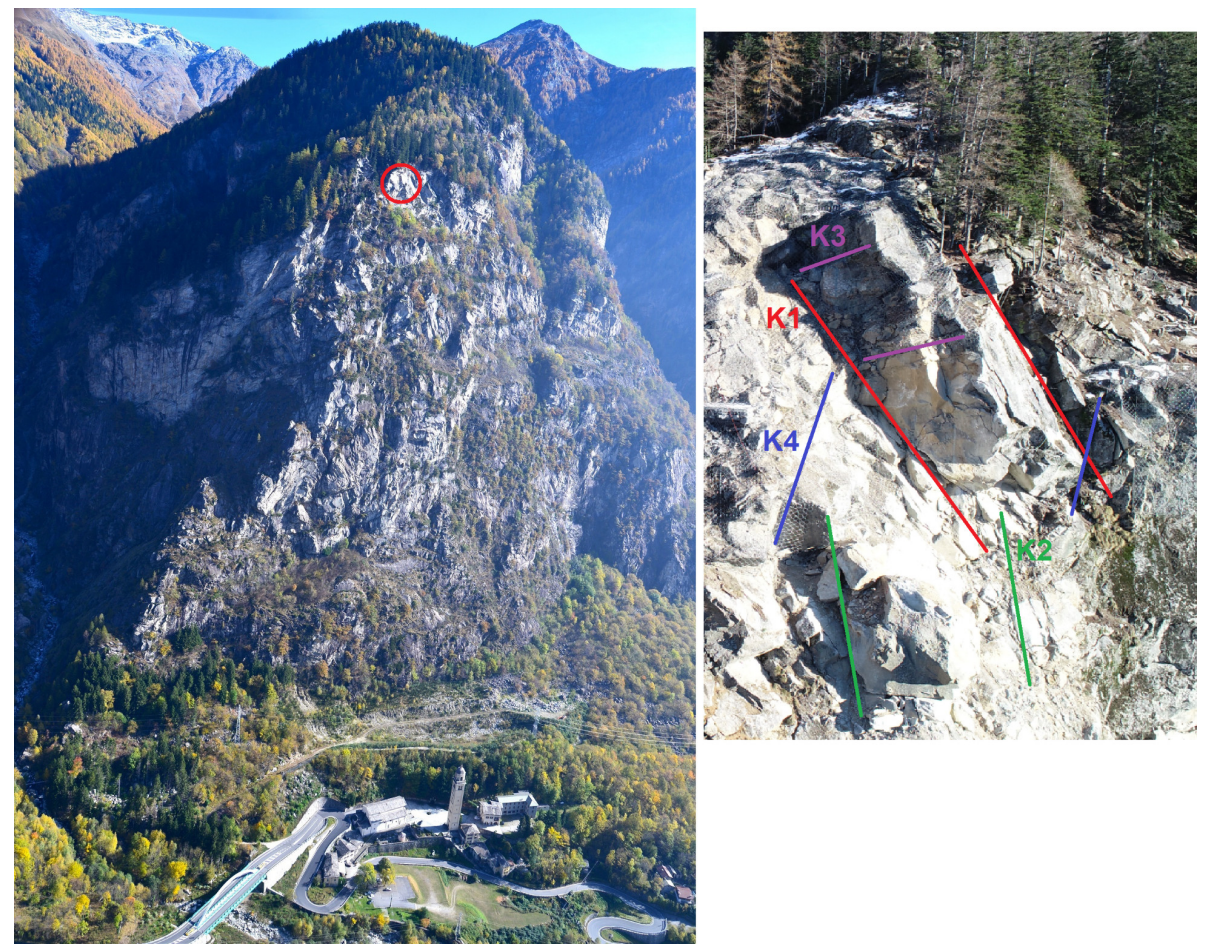

Figure 2. L'immagine a sinistra mostra la parete subverticale di Gallivaggio ai piedi della quale sono presenti le costruzioni afferenti al Santuario e la strada statale n. 36 per il Passo dello Spluga. Nel cerchio l'area da cui si è originato il crollo del 2018. La foto a destra mostra in dettaglio l'area di distacco dei blocchi rocciosi e i principali piani di discontinuità dell'ammasso.
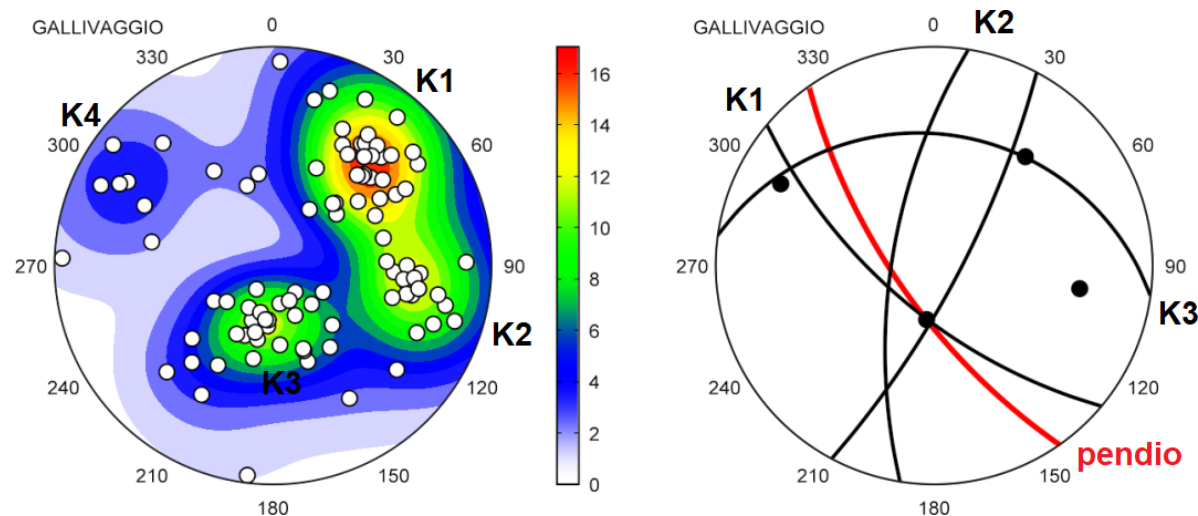

Figure 3. Rappresentazione stereografica (proiezione equiareale, emisfero inferiore) dei piani di frattura presenti nella porzione superiore della parete rocciosa di Gallivaggio. A sinistra sono mostrati i poli dei piani misurati, a destra le giaciture medie delle famiglie di discontinuità e del pendio $\left(235 / 70^{\circ}\right)$. Dall'analisi geometrica (Hoek e Bray, 1981) si ha conferma che il sistema K1 favorisce gli scivolamenti planari, come avvenuto nell'evento del maggio 2018.

l'ammasso roccioso franato a maggio del 2018, appartiene alla famiglia K1 (Fig. 3). L'evento ha comportato il distacco di una massa rocciosa (Dei Cas et al., 2018) valutata, grazie al confronto fra le nuvole di punti ottenute dalle immagini acquisite con drone prima e dopo l'evento franoso, complessivamente in circa $6700 \mathrm{~m}^{3}$ (Menegoni et al., 2020).

La parete dello Scaiùn, sita all'interno del territorio comunale di Val Masino (SO) in località Cataeggio (Fig. 4), dista in linea d'aria poche decine di $\mathrm{km}$ dalla zona di Gallivaggio. Geologicamente si trova nella parte sud orientale del Plutone terziario Masino Bregaglia, costituito prevalentemente da tonalite e granodiorite (Schmid et al., 1996).

Analogamente alla parete del Gallivaggio anche quella dello Scaiùn presenta al piede ampi coni di detrito in coalescenza fra loro e grossi blocchi rocciosi, testimonianza 


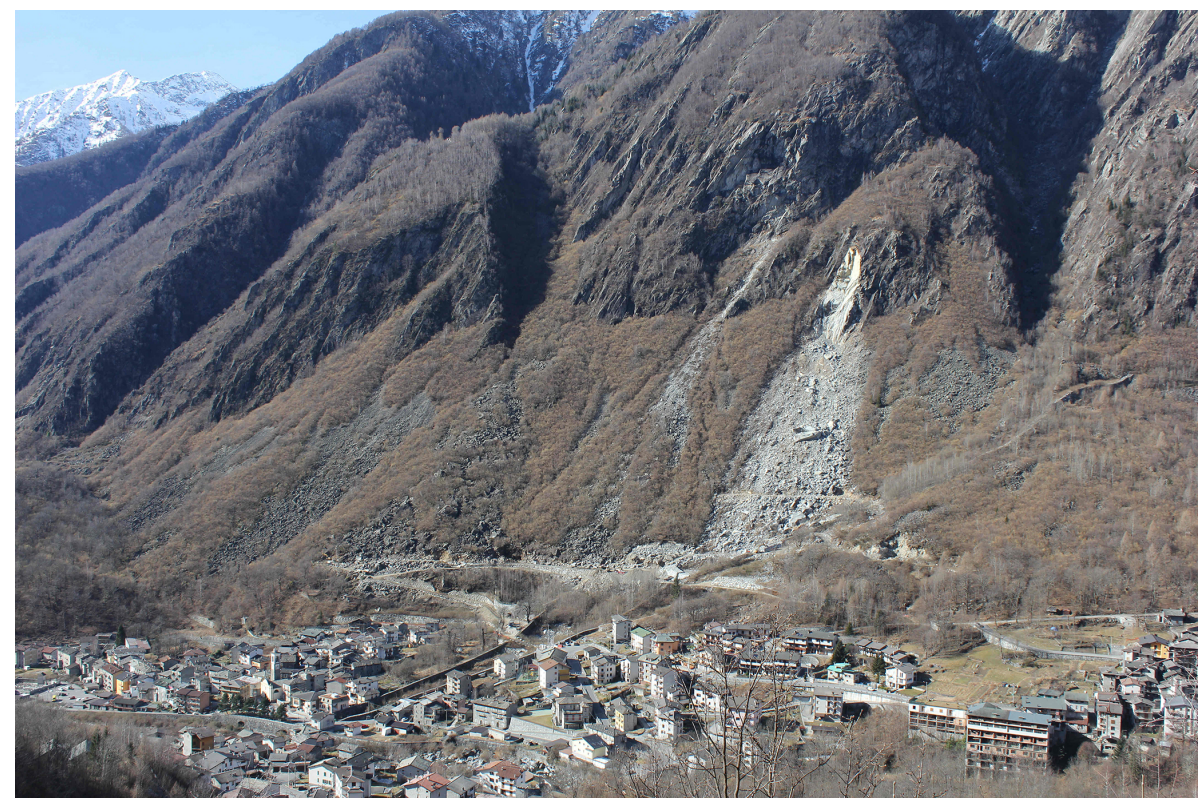

Figure 4. Nell'immagine, dall'alto verso il basso, si può osservare la parete dello Scaiùn, l'ampia falda di detrito all'interno della quale spicca, in quanto priva di vegetazione, l'area dei crolli più recenti e l'abitato di Cataeggio posto in sinistra e in destra idrografica del torrente Materlo.

di crolli pregressi. A dimostrazione di importanti eventi di crollo avvenuti in passato, il nucleo storico di Cataeggio presenta al proprio interno blocchi rocciosi decametrici (Fig. 5). Detti blocchi, costituiti quasi esclusivamente da tonalite (commercialmente nota come "Serizzo"), sono caratterizzati sia da facce delimitate da spigoli subarrotondati (a testimonianza dell'azione erosiva di fenomeni esogeni che hanno agito in passato) sia da spigoli vivi; questi ultimi si sono formati a seguito del distacco di blocchi di notevoli dimensioni che, crollando dalle pareti rocciose sovrastanti l'abitato, si sono frammentati in blocchi di minori dimensioni. La loro presenza è altresì compatibile con la litologia che caratterizza il margine meridionale del plutone del Masino-Bregaglia, dove appunto sorge l'abitato di Cataeggio.

Tra i principali sistemi di discontinuità, rilevati dall'analisi geomeccanica, sono presenti le famiglie K1 (giacitura media $\left.163 / 59^{\circ}\right)$ e $\mathrm{K} 2\left(165 / 86^{\circ}\right)$ che presentano la medesima immersione, ma inclinazione diversa, caratteristica ben evidente in affioramento. K1 risulta inoltre essere parallela ai piani di foliazione dell'ammasso e ai principali solchi vallivi presenti nell'area, quali la parte superiore della Val Materlo, della Val Pegolera e della Valle di Sasso Bisolo. Gli alti sistemi di discontinuità, che contribuiscono alla formazione di blocchi rocciosi isolati dall'ammasso, sono K3 $\left(69 / 86^{\circ}\right)$, K4 $\left(94 / 53^{\circ}\right)$ e K5 $\left(263 / 81^{\circ}\right)$. Il franamento avvenuto il 4 febbraio 2019 ha portato in luce un ampio piano di scivolamento dei sistemi K1 e K2, caratterizzanti l'intero versante sovrastante Cataeggio (Figs. 6 e 7).

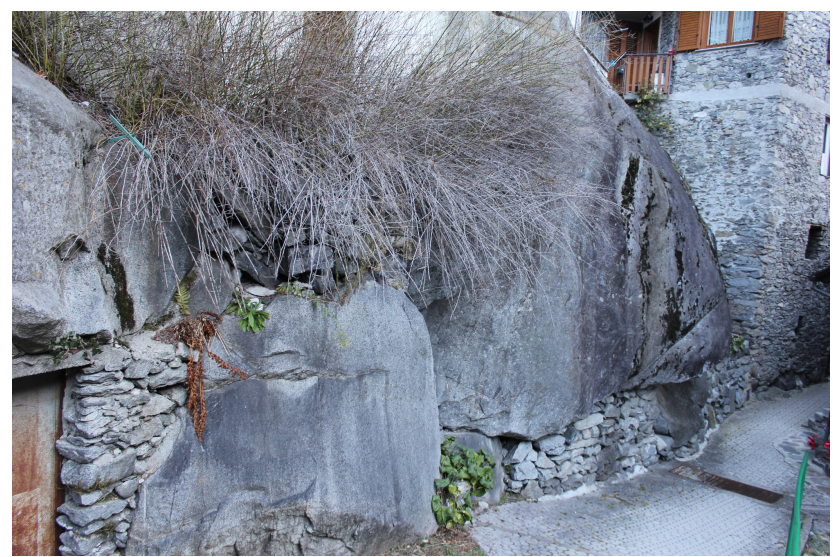

Figure 5. La parte storica dell'abitato di Cataeggio vede la presenza di numerosi massi ciclopici di Serizzo, sostanzialmente inglobati nelle costruzioni, a testimonianza dell'importanza dei fenomeni di crollo.

\section{Modifica delle condizioni di rischio delle aree}

Il rilievo geologico di massi e detriti presenti ai piedi delle pareti e ancor più le testimonianze degli eventi più recenti dimostrano che fenomeni di scivolamenti in roccia o crolli, provenienti dalle pareti di Gallivaggio e Scaiùn, si ripetono nel corso del tempo. Ciò ci permette di affermare che entrambe le aree sono soggette a una condizione di pericolo $(P)$ che è sostanzialmente immutata da anni (Varnes, 1984). 


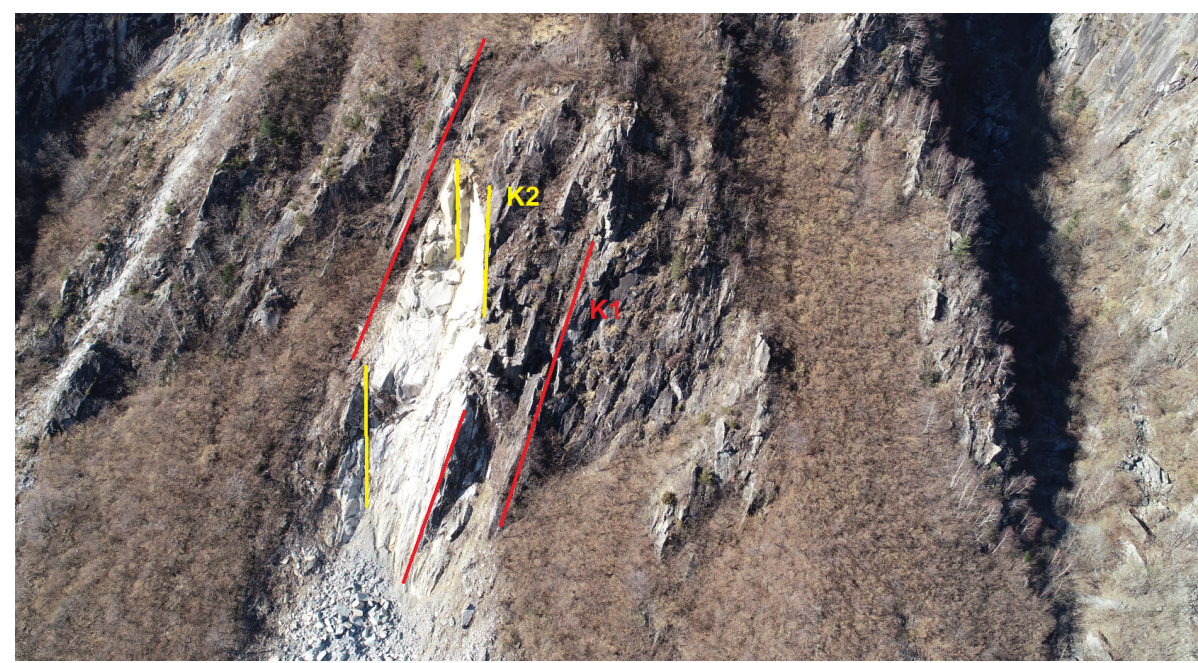

Figure 6. Dettaglio dell' area del distacco di febbraio 2019. I piani di discontinuità delle famiglie K1 $\left(163 / 59^{\circ}\right)$ e K2 (165/86 $\left.{ }^{\circ}\right)$ rappresentano i principali elementi di separazione dell' ammasso crollato dalle pareti retrostanti. Le altre famiglie individuate costituiscono essenzialmente gli elementi di svincolo laterale dei blocchi coinvolti nella caduta. La parete rocciosa da cui provengono i blocchi e le valli minori visibili nell'immagine sono orientate parallelamente al piano K1.
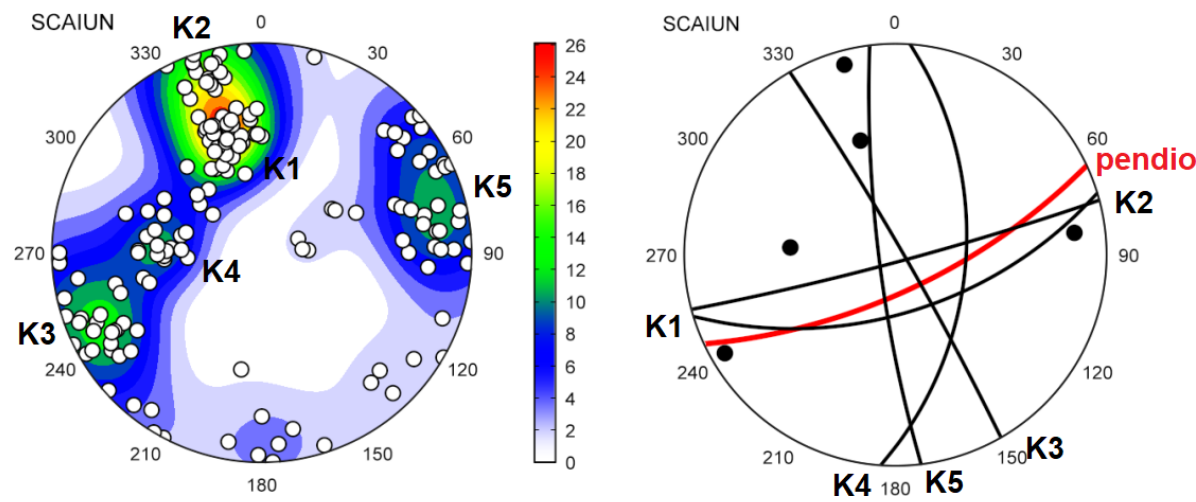

Figure 7. Rappresentazione stereografica (proiezione equiareale, emisfero inferiore) dei piani di frattura presenti lungo la parete rocciosa dello Scaiùn. A sinistra sono mostrati i poli dei piani misurati, a destra le giaciture medie delle famiglie di discontinuità e del pendio su cui è avvenuto il distacco nel febbraio 2019 (155/70). Dall'analisi geometrica (Hoek e Bray, 1981) si ha conferma che i sistemi K1 e K2 favoriscono gli scivolamenti planari di prismi rocciosi.

Non altrettanto può però dirsi per quanto riguarda il rischio $(R)$ che, nelle ultime decine di anni, è andato modificandosi sia in termini negativi, a causa delle mutate condizioni urbanistiche e viabilistiche (Fig. 8), sia in termini di riduzione dello stesso, grazie alla progettazione e messa in atto di numerosi interventi strutturali e non.

Per lo scopo che ci prefiggiamo non verranno di seguito analizzate le mutazioni urbanistico/viabilistiche, che hanno determinato l'aumento di vulnerabilità $(V)$ e valore $(W)$ delle zone esposte, mentre ci si si concentrerà sulle azioni volte alla riduzione del rischio stesso.

L'installazione di una rete di monitoraggio ha quale finalità quella di incidere sulla quantificazione del rischio. La diminuzione dello stesso, prodotta dall'installazione di un sistema di monitoraggio geologico con funzione di early warning, è possibile grazie alla riduzione dell'esposizione al pericolo di persone e cose; queste possono infatti essere tempestivamente evacuate dall'area in pericolo.

Con la medesima finalità di ridurre il livello di rischio, oltre alla rete di monitoraggio, sulle aree oggetto di studio sono state realizzate numerose opere di difesa, visibili anch'esse nella Fig. 8.

Per quanto riguarda l'area di Gallivaggio, la messa in opera di significative opere di difesa ha avuto inizio nei primi anni del XXI secolo con la posa in opera di reti di trattenuta, quindi con un rilevato in terra (2006) la cui azione di protezione è stata amplificata ponendo una rete paramassi all'apice e sul fianco destro dello stesso (2008). Successivamente una ulteriore difesa passiva (rete di trattenuta) è stata posta da ANAS (Azienda Nazionale 


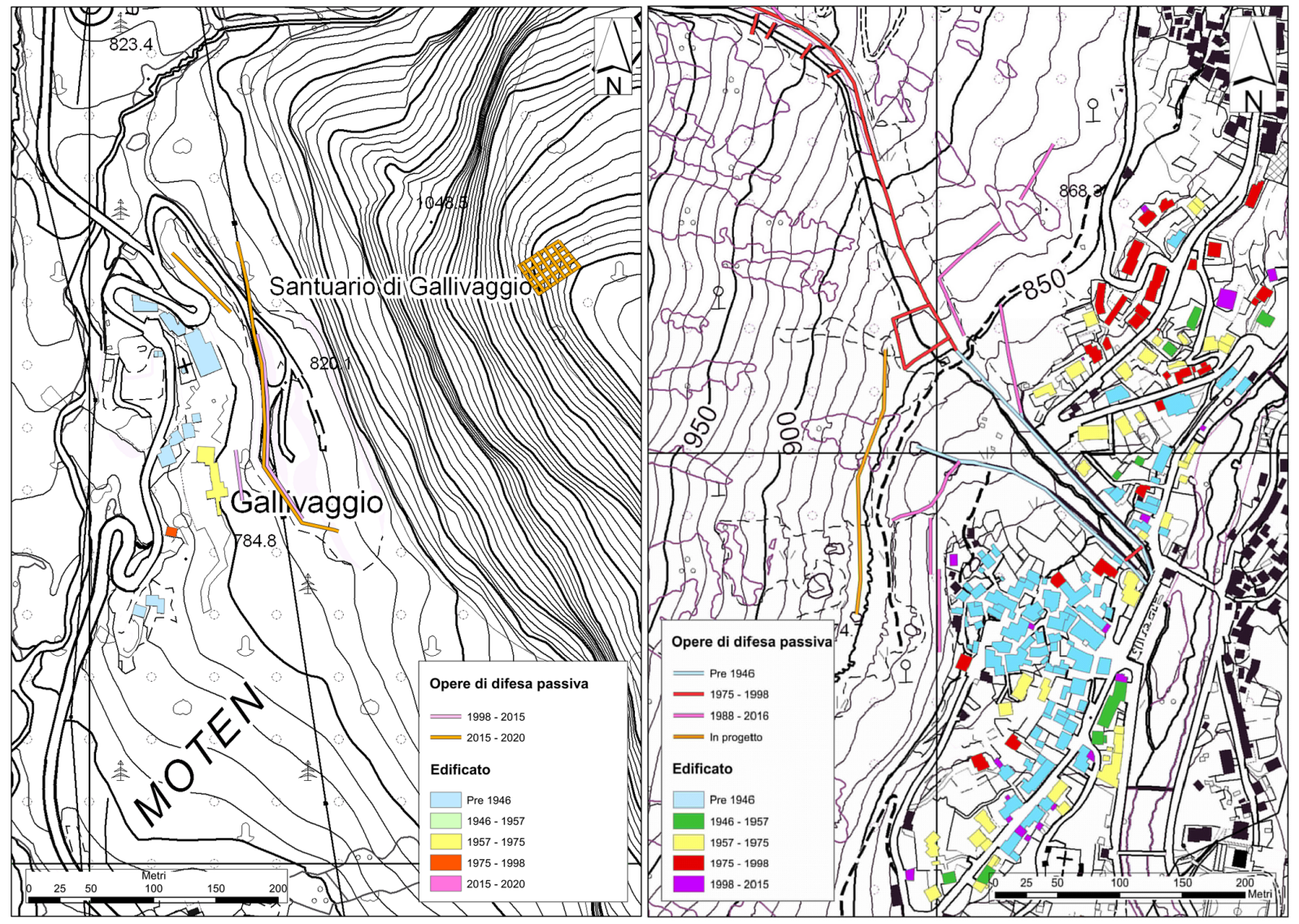

Figure 8. Attestando la nostra analisi sul periodo post-bellico possiamo osservare come l'edificato e quindi verosimilmente la presenza di persone sia aumentata soprattutto nel periodo 1957-1998. Avendo effettuato da foto aerea il rilievo delle costruzioni è possibile che non tutto l'edificato rilevato corrisponda a edifici destinati alla presenza stabile di persone. Gallivaggio nella figura di sinistra, Cataeggio in quella di destra. (Base cartografica - Carta Tecnica Regionale di Regione Lombardia: licenza CC-BY-NC-ND 4.0.)

Autonoma delle Strade) a protezione della strada statale. Recentemente, sia prima sia dopo l'evento franoso del 2018, sono state eseguite anche opere di difesa nella parte più rilevata della parete ed è in corso il rifacimento del vallo paramassi a protezione dell'area del Santuario (Cancelli et al., 2018).

Nell'area di Cataeggio negli ultimi 30 anni gli interventi sono stati numerosi e volti sia alla difesa delle colate detritiche provenienti dalla Val Materlo (a completamento di quanto realizzato successivamente all'evento alluvionale dell'agosto 1911) sia alla difesa da crolli. Prima del 1988 nell'area di Cataeggio l'unica difesa presente era costituita dai due argini posti lungo l'alveo del torrente Materlo, in corrispondenza dell'antico centro abitato. Successivamente sono state realizzate alcune opere di difesa passiva, a tutela degli edifici presenti sia in destra sia in sinistra del torrente (Del Simone, 2016a; Grossi, 2016a; Songini, 2010, 2011). Anche in questo caso è attualmente in corso d'esecuzione un vallo in terra armata a difesa dell'abitato.
Attualizzando, mediante l'indice ISTAT dei prezzi al consumo, gli importi necessari per l'esecuzione di queste opere, si può constatare che su Gallivaggio e Cataeggio, negli ultimi trent' anni, sono stati complessivamente stanziati oltre 10 milioni di euro per interventi strutturali distribuiti pressoché omogeneamente (c'è una modesta prevalenza di Gallivaggio) fra le due aree come visibile nella Tabella 1.

\section{Monitoraggio interferometrico delle aree: descrizione dei sistemi e costi}

Entrambi i dissesti oggetto di studio sono stati monitorati con il medesimo modello di radar da terra ad apertura sintetica (GBInSAR LiSALab) e per entrambe le aree si è passati da una prima fase di monitoraggio periodico, con misure puntuali distanziate di uno o più mesi, a un monitoraggio continuo in near real time.

$\mathrm{Su}$ entrambe le aree sono state calcolate precisioni, sulle mappe di spostamento ottenute dalle immagini radar acquisite, e accuratezze di decimi di millimetri. 
Table 1. Interventi eseguiti negli ultimi decenni nelle aree di Cataeggio (tabella sopra) e Gallivaggio.

\begin{tabular}{|c|c|c|c|}
\hline Anno & CATAEGGIO: tipo di intervento & $\begin{array}{l}\text { Ente } \\
\text { attuatore }\end{array}$ & $\begin{array}{r}\text { Importo } \\
\text { economico } \\
\text { (arrotondato EUR) }\end{array}$ \\
\hline $1988-1990$ & Argine sinistro Val Materlo & R. Lomb. & 576000.00 \\
\hline \multirow[t]{2}{*}{$1994 / 1998$} & Arginatura e sacca L. 102/90 & R. L./CM & 835000.00 \\
\hline & Vallo in muratura a protezione crolli & & 250000.00 \\
\hline 2011 & Rilevati in terra sinistra idrografica a protezione abitati & Comune & 150000.00 \\
\hline 2016 & Rilevato paramassi scogliera in sinistra Val Materlo & Comune & 80000.00 \\
\hline 2017 & Viabilità e reti paramassi sopra l'abitato & Comune & 300000.00 \\
\hline 2018 & Seconda vasca su Materlo e rilevato paramassi argine sinistro & Comune & 100000.00 \\
\hline 2020 & Svaso torrente, potenziamento argine sinistro ed opera trattenuta & Comune & 150000.00 \\
\hline 2020 & Vallo in terra armata (in esecuzione) & Comune & 2600000.00 \\
\hline 2020 & Manutenzione alveo Materlo (in progettazione) & Comune & 100000.00 \\
\hline Anno & GALLIVAGGIO: tipo di intervento & $\begin{array}{l}\text { Ente } \\
\text { attuatore }\end{array}$ & $\begin{array}{r}\text { Importo } \\
\text { economico } \\
\text { (arrotondato EUR) }\end{array}$ \\
\hline 2004 & Pronto intervento & Comune & 30000.00 \\
\hline 2006-2007 & Realizzazione vallo e rilevato in terra & Comune & 600000.00 \\
\hline 2008 & Pronto intervento: installazione reti paramassi all'apice del rilevato in terra & Comune & 200000.00 \\
\hline 2015 & Barriera elastoplastica da $2000 \mathrm{~kJ}$ & ANAS & 110000.00 \\
\hline 2017 & Rete in parete e disgaggio & $\mathrm{CM}$ & 70000.00 \\
\hline 2018-2019 & Rete in aderenza e disgaggio & $\mathrm{CM}$ & 1300000.00 \\
\hline 2020 & Nuova barriera in terra armata e rete di trattenuta (in esecuzione) & $\mathrm{CM}$ & 3300000.00 \\
\hline
\end{tabular}

Per completezza si ricorda che nell'area di Gallivaggio vengono periodicamente effettuate anche misure distometriche manuali a controllo dei movimenti di alcune fratture.

Per quantificare i costi di gestione dei sistemi di monitoraggio è necessario considerare non solo il costo del servizio di rilevamento con radar da terra e trasmissione dei dati, ma tenere in debito conto sia tutti quei costi che non vengono effettuati annualmente (vedi ad esempio gli studi di modellazione, i costi di installazione del sistema o gli allacci elettrici) sia quelli necessari per una completa gestione della rete di monitoraggio (quali i costi di uffici, strutture, strumentazione, personale dedicato per misure manuali e analisi dei dati in tempo reale).

Per detta quantificazione sono stati utilizzati i costi di gestione del Centro di Monitoraggio Geologico di ARPA Lombardia su un arco temporale di dieci anni, così da poter conteggiare in maniera bilanciata anche le attività periodiche. Il risultato ci permette di stimare in circa EUR 700000 l'importo necessario per gestire, per un periodo di 10 anni, le reti di monitoraggio di Gallivaggio o Cataeggio.

\section{Descrizione evento Gallivaggio 2018 e Cataeggio 2019}

Durante la primavera 2018, sulla parete del Gallivaggio, si sono manifestati importanti movimenti che hanno portato al franamento di $6700 \mathrm{~m}^{3}$ il 29 maggio 2018 (Menegoni et al., 2020). Questo evento è solo l'ultimo di una serie di franamenti di cui si trova traccia nella documentazione storica, vedasi a titolo di esempio il crollo del 1660 di un grosso masso da cui si ricavò il portale maggiore del Santuario (Scaramellini, 1998), o che possono essere ricostruiti in quanto piuttosto recenti e avvenuti più volte anche negli ultimi anni. In concomitanza dell'ultimo di questi eventi, avvenuto il 13 aprile 2018, un ampio settore di versante roccioso ha iniziato a far registrare movimenti superiori alle soglie prestabilite ( $3 \mathrm{e} 4 \mathrm{~mm}$ in $24 \mathrm{~h}$ ) tanto che, a seguito dei dati registrati con il monitoraggio, le abitazioni sottostanti sono state evacuate; il Santuario Mariano, edificato fra la fine del XVI secolo e l'inizio del XVII, è stato reso inagibile asportando preventivamente buona parte delle opere sacre presenti. La strada statale dello Spluga è stata chiusa al traffico o, in occasione di movimenti meno accentuati, aperta solo per alcune ore al giorno. A partire dal 24 maggio, data in cui è stata irreversibilmente superata la soglia di $4 \mathrm{~mm}$ in $24 \mathrm{~h}$, la circolazione stradale è stata completamente interrotta.

Le giornate che hanno preceduto il crollo del 29 maggio hanno fatto registrare un notevole incremento degli spostamenti (compresi fra 18 e $32 \mathrm{~cm}$ in 10 giorni) dell'ammasso roccioso, come facilmente osservabile dai dati di spostamento di punti interferometrici ubicati sulla parte di versante poi crollato (Fig. 9) (Dei Cas et al., 2018). Va sottolineato che, soprattutto per le ultime ore antecedenti il franamento, i dati sono stati sottoposti a processo di srotolamento della fase (unwrapping) al fine di 


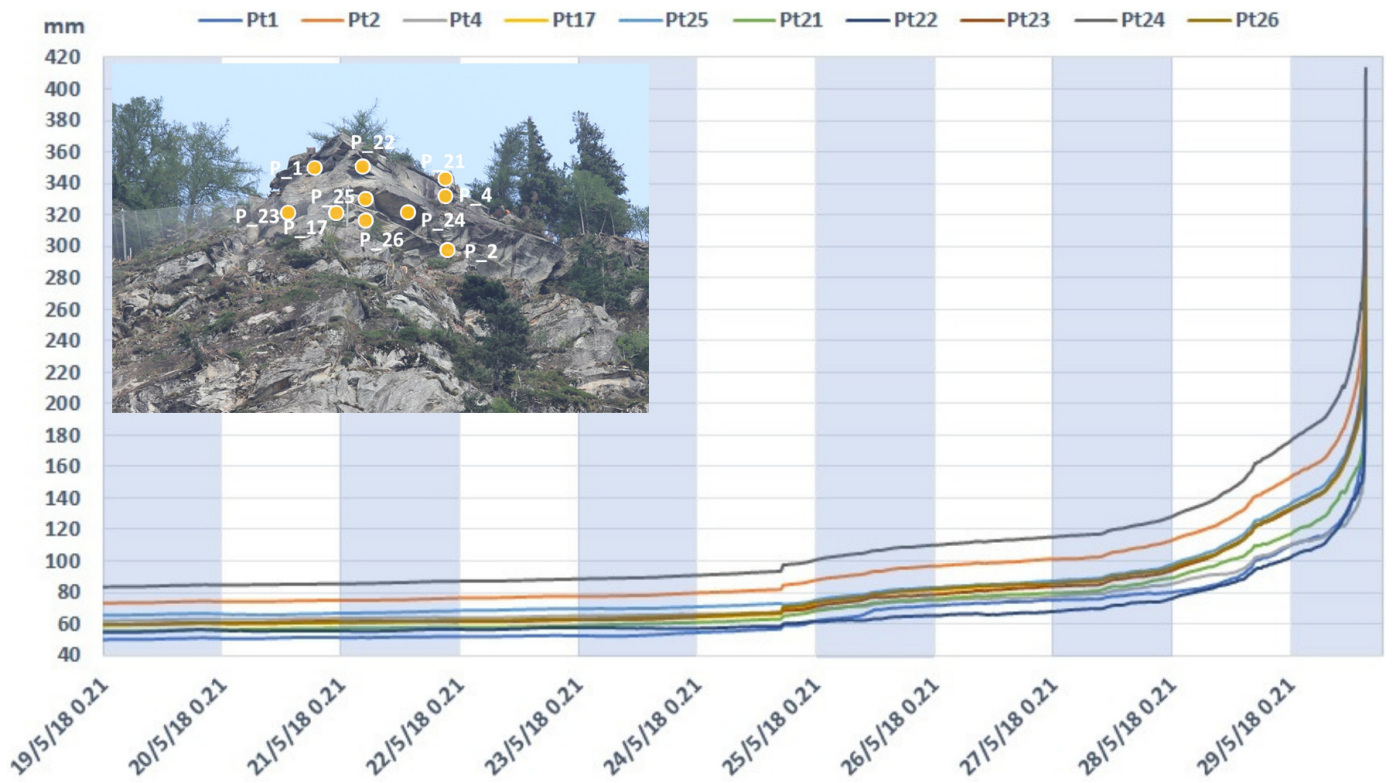

Figure 9. Spostamenti misurati al Gallivaggio dal 19 al 29 maggio, vale a dire gli ultimi dieci giorni prima del franamento, lungo la linea di vista del radar (LOS) di alcuni punti virtuali posti sull'area in movimento della parete rocciosa.

definirne il valore corretto in quanto, anche l'esecuzione di interferogrammi con immagini acquisite a distanza di circa $2^{\prime} 30^{\prime \prime}$, evidenziava valori superiori, a volte molto superiori, a 0.25 della lunghezza d'onda utilizzata dal radar.

Alle ore 16:35 LT circa del 29 maggio 2018, 6700 metri cubi di roccia si sono staccati dalla parte sommitale della parete di Gallivaggio e sono precipitanti andando a interessare una ampia zona di fondovalle come evidenziata in Fig. 10.

Per quanto riguarda l'area di Cataeggio la ricostruzione degli eventi non può vantare una documentazione storica plurisecolare, come quella di cui si dispone per il Santuario di Gallivaggio, ma gli eventi di cui si ha traccia nel XX secolo (Barbolini, 2018; Del Simone, 2016b; Grossi, 2016b; Songini, 2010, 2011; Erba, 1988) e soprattutto i grossi blocchi rocciosi presenti sul fondovalle possono confermare la ricorrenza di fenomeni di dissesto idrogeologico. A tal proposito a partire dall'evento del 1911, caratterizzato da fenomeni di colate detritiche, l'area è stata più volte soggetta a crolli e, con area di distacco in Val Materlo, si ha traccia anche di un fenomeno valanghivo che, intorno al 1900, ha raggiunto il torrente Masino (Songini, 2000).

Per quanto riguarda gli avvenimenti degli ultimi anni, sono state disposte evacuazioni sia per gli edifici residenziali sia per l'edificio scolastico (scuola elementare e asilo), ma sempre successivamente all'accadimento dei fenomeni di franamento.

Anche per l'area di Cataeggio è stato possibile ricostruire l'andamento, individuato con il radar da terra, di alcuni punti interferometrici posti sul versante franato il 4 febbraio 2019 e, come per l'area di Gallivaggio, si può osservare (Fig. 11),

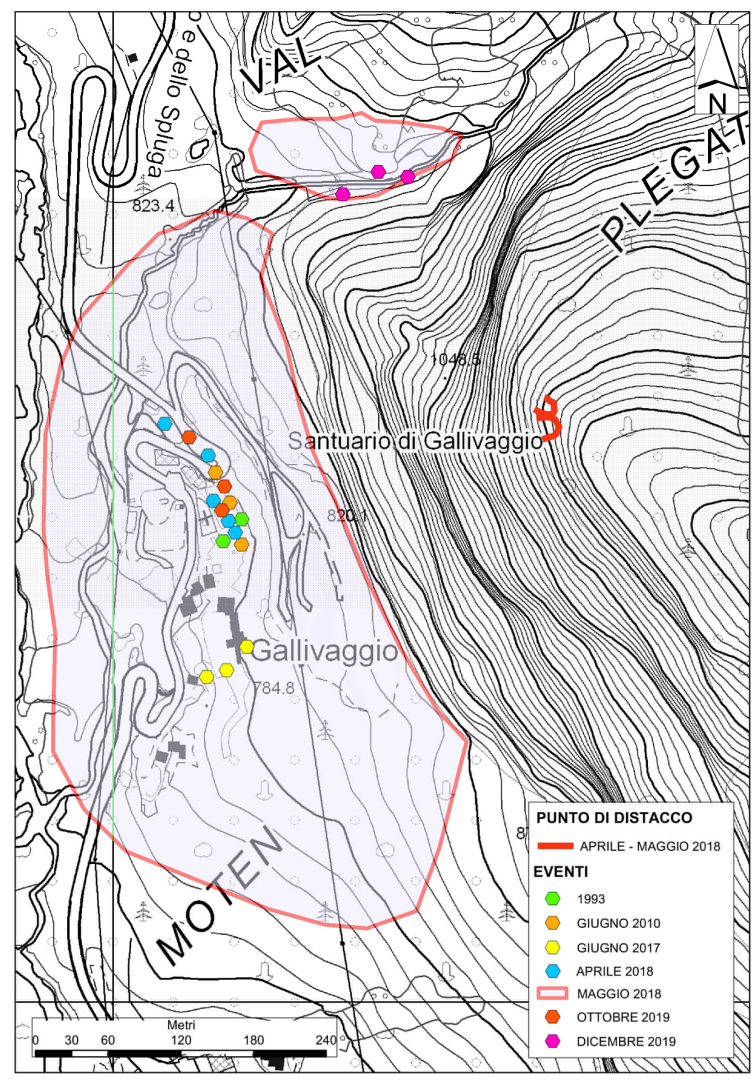

Figure 10. Cartografia relativa agli eventi di franamento e crollo più recenti. (Base cartografica - Carta Tecnica Regionale di Regione Lombardia: licenza CC-BY-NC-ND 4.0.) 


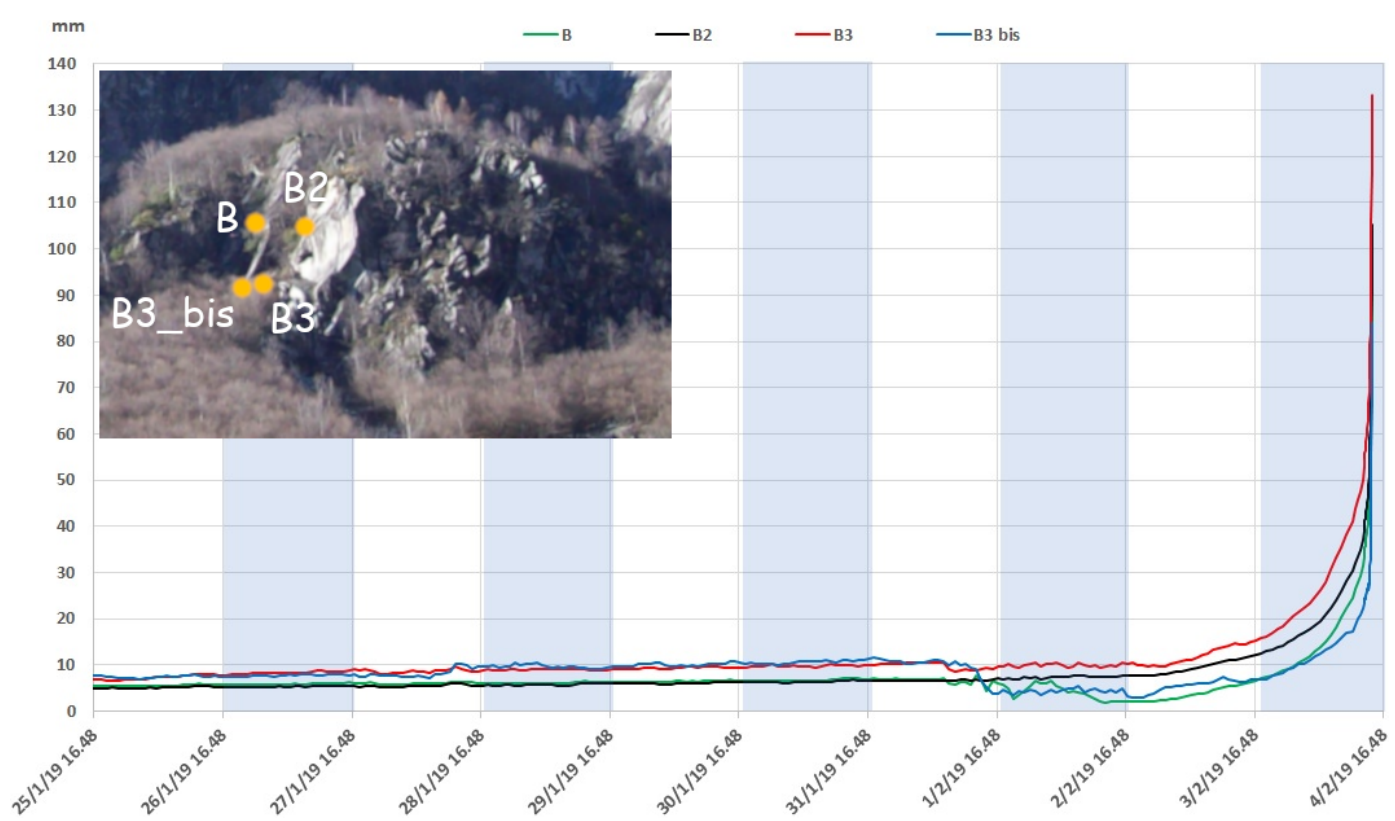

Figure 11. Spostamenti misurati a Cataeggio dal 25 gennaio al 4 febbraio ossia negli ultimi dieci giorni prima del franamento, lungo la linea di vista del radar (LOS) di alcuni punti virtuali posti sull'area in movimento della parete rocciosa.

dopo l'esecuzione del processo di srotolamento della fase, la forte evoluzione del movimento prima del crollo (spostamenti totali compresi fra 7 e $13 \mathrm{~cm}$ ).

In tale occasione non avendo preventivamente predisposto studi approfonditi, relativi al comportamento dell'ammasso roccioso della parete di Cataeggio, che permettessero la definizione di soglie d'allertamento (soglie sito specifiche), non erano state predeterminate procedure di allerta collegate al sistema di monitoraggio. In ragione di ciò l'attenzione sull'area è aumentata, con l'aumentare della velocità di movimento, ma le attività sono state circoscritte esclusivamente ad approfondimenti di carattere conoscitivo. A un paio d'ore dal crollo, ancorché l'ammasso roccioso si fosse mosso nell'ultime 12 ore ore di $2-3 \mathrm{~cm}$ ed evidenziasse ormai velocità orarie di $2-3 \mathrm{~mm}$, non era stato preso alcun provvedimento di carattere restrittivo relativamente a viabilità o presenza di persone in edifici pubblici o privati. Tali provvedimenti sono certamente presenti successivamente al crollo avvenuto alle 14:40 LT del 4 febbraio 2019. La distribuzione del materiale franato, di questo e di alcuni episodi precedenti, è riportata in Fig. 12.

\section{Discussione}

Come già accennato, quanto avvenuto nell'area di Gallivaggio e Cataeggio oltre a una prossimità temporale (maggio 2018-febbraio 2019) mostra numerose analogie. Anzitutto i franamenti hanno avuto origine da contesti geomorfologici analoghi, ossia da pareti rocciose subverticali, costituite rispettivamente da ortogneiss e quarzodiorite, che storicamente hanno fatto registrare fenomeni di dissesto.

Proprio tale reiterazione dei dissesti ha portato su entrambi $i$ siti, in diversi e successivi interventi, alla realizzazione di numerose opere di difesa passive, ossia quegli interventi "che agiscono rallentando, deviando od ostacolando la caduta, il rotolamento e il movimento di masse detritiche" (APAT 2002), a tutela dei sottostanti ambiti antropizzati. I due eventi, avvenuti a pochi mesi di distanza, possono essere entrambi classificati come scivolamenti traslazionali in roccia (Varnes, 1978). Hanno coinvolto quantitativi di materiale che, sulla base di modelli digitali del terreno (DTM) realizzati mediante tecniche aerofotogrammetriche da rilievi con drone, sono risultati di $6700 \mathrm{~m}^{3}$ nel primo caso e di $7900 \mathrm{~m}^{3}$ per Cataeggio.

A tutto ciò si deve aggiungere che, al momento del franamento, su entrambi i siti era funzionante un sistema di monitoraggio in near real time gestito mediante tecnica interferometrica con radar da terra ad apertura sintetica. Tale tecnica appare particolarmente adatta al monitoraggio di pareti rocciose, dove non ci sono evidenze superficiali della presenza di un piano di scivolamento e pertanto non è possibile agire con strumentazione tradizionale (p.e. mire ottiche e/o estensimetri). Il dato acquisito con l'interferometria radar permette infatti di ottenere informazioni in continuo sull'intera parete, permettendo l'attivazione dell'allarme su qualsiasi porzione di essa si vengano a evidenziare movimenti significativi.

Infine, osservando le curve di spostamento riportate nelle Figs. 9 e 11, è immediato osservare come le aree mostrino un andamento degli spostamenti del tutto analogo; entrambe 


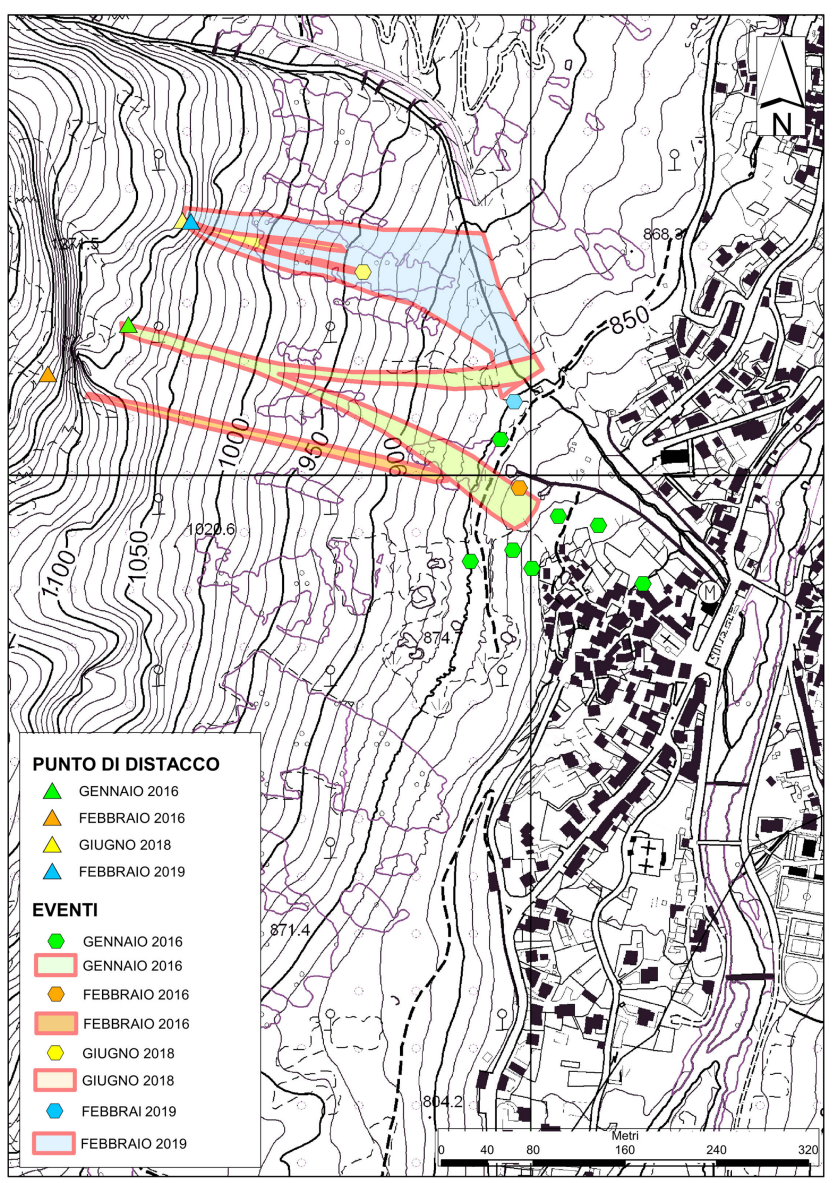

Figure 12. Cartografia degli areali coinvolti negli eventi più recenti (2016-2019). I poligoni all'esterno delle aree colorate individuano i punti di arrivo più prossimi alle abitazioni di alcuni massi singoli. (Base cartografica - Carta Tecnica Regionale di Regione Lombardia: licenza CC-BY-NC-ND 4.0.)

evolvono nel tempo fino a rottura tendendo all'asintoto verticale, caratteristica tipica di un creep terziario con comportamento viscoso quale quello che qui si riscontra.

Quest'ultima osservazione appare interessante per confermare le potenzialità di una rete di early warning posta a presidio di scivolamenti traslazionali su pareti rocciose dove, viceversa, la problematica di crolli improvvisi e istantanei non ha, a tutt'oggi, un efficace e sperimentato metodo di monitoraggio con funzione di early warning.

Rappresentate le analogie è necessario evidenziare le differenze tra $\mathrm{i}$ due eventi a cominciare da una analisi delle curve delle velocità medie orarie precedenti la rottura (Fig. 13) che, come visibile, si sviluppano con andamento analogo, ma con tempistiche e valori differenti.

Valutando l'evoluzione, per ogni elaborazione interferometrica, della velocità media oraria dei singoli punti a partire dal raggiungimento della velocità media giornaliera di $3 \mathrm{~mm}$ in $24 \mathrm{~h}$ (valore della soglia di preallarme per Gallivaggio), anzitutto è immediatamente riscontrabile come, fra il raggiungimento dei $3 \mathrm{~mm}$ in $24 \mathrm{~h}$ e il momento del crollo, siano intercorsi intervalli temporali estremamente differenti (27 ore per Cataeggio contro 126 per Gallivaggio). Ciò, tenendo conto delle azioni da intraprendere in occasione di emergenze di protezione civile, ha implicazioni non certo secondarie. Il confronto fra le due curve permette inoltre di apprezzare le velocità raggiunte che, come per gli spostamenti di cui alle Figs. 9 e 11, presentano valori differenti.

Di per sé il fatto che si manifestino valori differenti (spostamenti o velocità) potrebbe essere determinato anche solo dalle differenti linee di vista (LOS) dei radar rispetto alla direzione principale di movimento della frana, ma una valutazione sui rapporti delle accelerazioni $(R)$, nei medesimi intervalli di tempo, permette di escludere questa ipotesi.

In particolare questo è evidente nel rapporto fra le accelerazioni medie orarie di un singolo punto in predefiniti intervalli di tempo a partire da un tempo $t_{0}$, inteso come l'ultima accelerazione oraria disponibile prima del crollo, fino ad un tempo $t_{12}$ inteso come l'accelerazione media oraria 12 ore (Fig. 14) prima del franamento. Il valore delle accelerazioni dei punti interferometrici è simile se confrontato con i punti della medesima area (p.e. mediamente le accelerazioni dell'ultima ora dei tre punti di Gallivaggio è intorno al valore di $103 \mathrm{~mm} \mathrm{~h}^{-2}$ mentre quello dei tre punti di Cataeggio si pone attorno a $55.5 \mathrm{~mm} \mathrm{~h}^{-2}$ ).

I rapporti fra le accelerazioni $(R)$ assumono valore costante $(R \approx 11)$ solo nell'ultimo caso (Fig. 14a) mentre evidenziano elevata variabilità negli altri intervalli. Per quanto riguarda il crollo di Gallivaggio possiamo rilevare valori progressivamente in crescita da circa $6 \mathrm{~mm} \mathrm{~h}^{-2}$ a oltre $100 \mathrm{~mm} \mathrm{~h}^{-2}$, a testimonianza di una accelerazione continua e progressiva nelle ultime 12 ore. Per quanto riguarda Cataeggio il dato evidenzia valori da prossimi all'unità a circa $60 \mathrm{~mm} \mathrm{~h}^{-2}$, a significare che solo nelle ultime 3 ore l'accelerazione ha assunto valori significativi.

Queste differenze comportamentali, legate all'incremento della velocità, possono essere ulteriormente osservate mediante le classiche curve dell'inverso della velocità, tipiche dei creep terziari (Fukuzono, 1985; Voight, 1989), dove la previsione del tempo di accadimento del dissesto è determinata dall'intersezione della retta $1 \mathrm{v}^{-1}$ (con $\mathrm{i}$ dati di velocità ricavati dai valori dei punti interferometrici) con l'asse temporale (Fig. 15). È possibile osservare come i dati di Gallivaggio evidenzino una tendenza che sin da almeno 4 ore prima del franamento converge verso una retta, con coefficiente angolare costante, indirizzata a intersecare l'asse del tempo in $\mathrm{tf}$ (tempo di franamento). Viceversa, per Cataeggio la convergenza dei punti avviene in un tempo assai più prossimo (tra $1 \mathrm{~h}$ e $40 \mathrm{~min}$ e $1 \mathrm{~h}$ e $15 \mathrm{~min}$ ) al franamento.

Oltre ai differenti tempi di evoluzione dell'evento naturale è importante anche rappresentare le diverse modalità di approccio nella gestione del rischio a partire dalle misure 


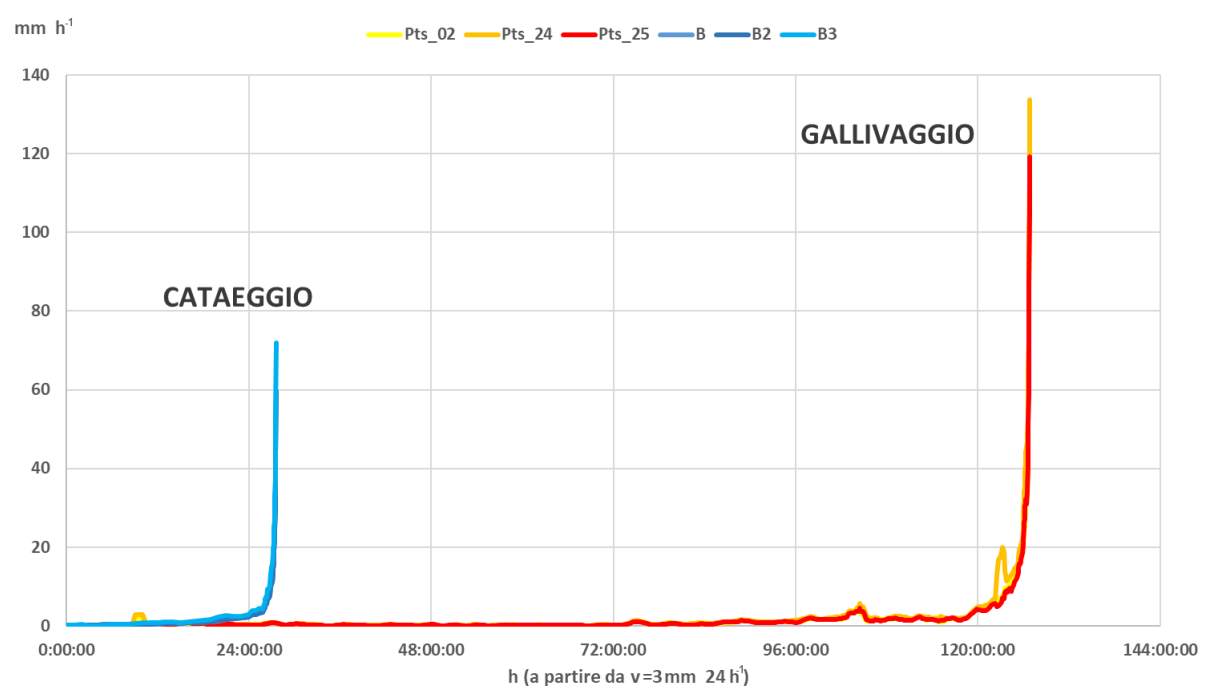

Figure 13. Curve di velocità media oraria dei punti virtuali rappresentativi dei due versanti in movimento.

di evacuazione preventiva: a Gallivaggio, detta evacuazione preventiva, venne disposta, sulla base di una modellazione del fenomeno franoso e di soglie di velocità giornaliere preventivamente fissate, sin dall'inizio dell' evidenziarsi della criticità. A Cataeggio, viceversa, ancora poche ore prima del crollo, nonostante un'ampia area di versante fosse in movimento con velocità superiori a $2 \mathrm{~mm} \mathrm{~h}^{-1}$, non erano stati presi provvedimenti di protezione civile circa il traffico veicolare o la presenza di persone in edifici scolastici e privati.

A Gallivaggio la presenza di soglie d'allertamento prefissate permette di osservare una efficacia $(E)$ dei sistemi di early warning molto elevata. Ciò è facilmente dimostrabile in quanto misure di evacuazione preventiva o permanenti limitazioni al traffico veicolare non erano mai state messe in atto prima degli eventi della primavera 2018.

Ulteriori differenze si possono riscontrare anche nella gestione territoriale come visibile dall'evoluzione urbanistica delle aree a rischio (Fig. 8): nel caso di Gallivaggio l'incremento edilizio degli ultimi 70 anni corrisponde sostanzialmente alla sola Casa del Pellegrino e l'aumento delle condizioni di rischio è determinato per lo più dall'incremento del traffico veicolare. Per quanto riguarda Cataeggio, a parità di incremento del traffico veicolare, si può stimare sia avvenuto un ben più cospicuo incremento delle condizioni di rischio determinato dall'edificazione che, a partire dal 1956, è stata importante.

Su entrambe le aree è immediatamente osservabile come, successivamente all'aumento del rischio determinato da un incremento di vulnerabilità e valore delle zone esposte, siano state messe in atto opere di difesa passiva concretizzatesi in più interventi.

Analogamente l'avvio di una rete di monitoraggio (misura non strutturale di riduzione del rischio) nelle aree di Gallivaggio (2011) e Cataeggio (2016) è iniziata a seguito di eventi franosi che hanno raggiunto o sono arrivati in prossimità delle aree antropizzate.

\section{Conclusione}

È conoscenza ormai comune e consolidata che, al mutare delle condizioni sociali avvenute per lo più nel $\mathrm{XX}$ secolo, sia corrisposta una più o meno accentuata variazione delle condizioni di rischio naturale gravante su un determinato territorio. Ciò è ovviamente valido anche per alcune aree poste a ridosso di pareti rocciose subverticali come quelle oggetto di studio. Tale situazione, unitamente a una differente percezione del rischio e del concetto di sicurezza, ha fatto sì che i decisori pubblici realizzassero importanti interventi, volti alla realizzazione di opere di difesa, ma anche allo sviluppo di reti di monitoraggio geologico con valenza di early warning.

In tale contesto i fenomeni franosi, avvenuti a Gallivaggio e Cataeggio, hanno evidenziato come l'interferometria con radar da terra risulti una metodologia particolarmente indicata e affidabile nel monitoraggio, con finalità di protezione civile, delle pareti rocciose. In detti ambiti ci si trova a dover indagare fenomeni estremamente lenti (Cruden e Varnes, 1996) che più o meno repentinamente possono assumere velocità anche di parecchi centimetri al giorno.

È quindi indispensabile disporre di strumentazione di misura altamente performante che possa registrare normalmente movimenti giornalieri inferiori al decimo di millimetro e che però, con altrettanta precisione, possa gestire anche movimenti giornalieri di tre ordini di grandezza superiori. In aggiunta l'interferometria con radar da terra appare una metodologia particolarmente indicata per restituire una informazione areale, laddove non si possa individuare con precisione la frattura o il lineamento dove si localizzerà il distacco, come nel caso delle pareti oggetto di 
(a)

Rapporto di accelerazione A0-1/A1-3

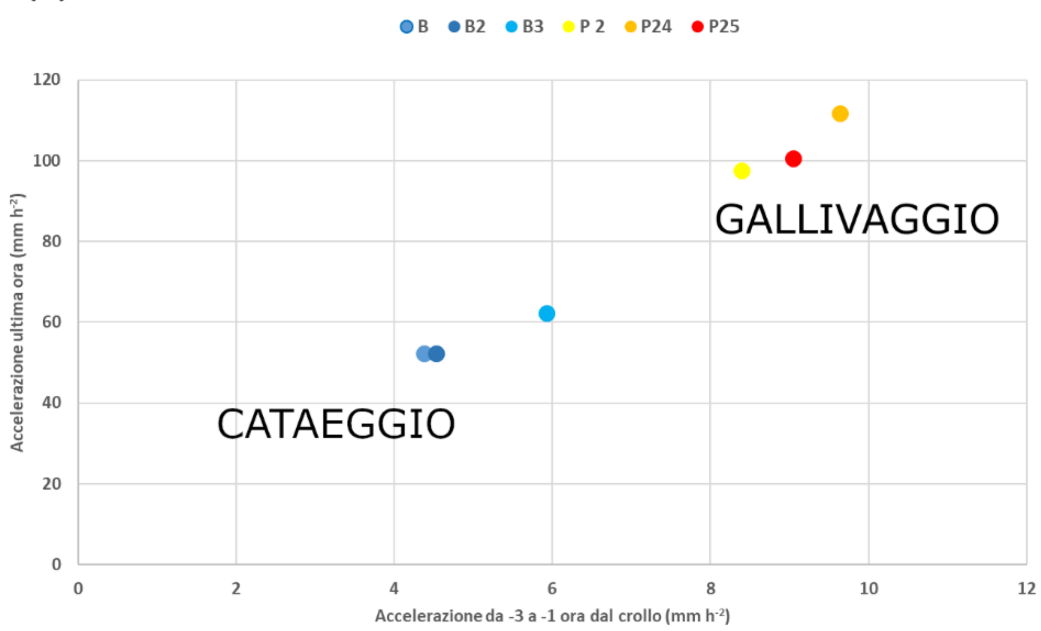

(b)

Rapporto di accelerazione A1-3/A3-6

$\bullet$ B $\bullet$ B2 $\bullet$ B $\quad$ P $2 \bullet$ P24 $\bullet$ P25

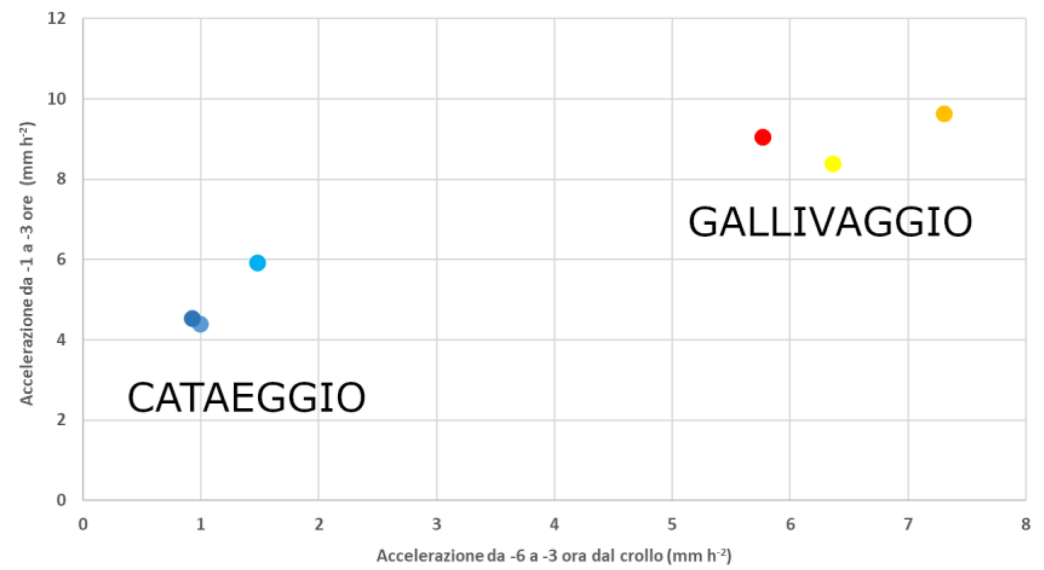

(c)

Rapporto di accelerazione A3-6/A6-12

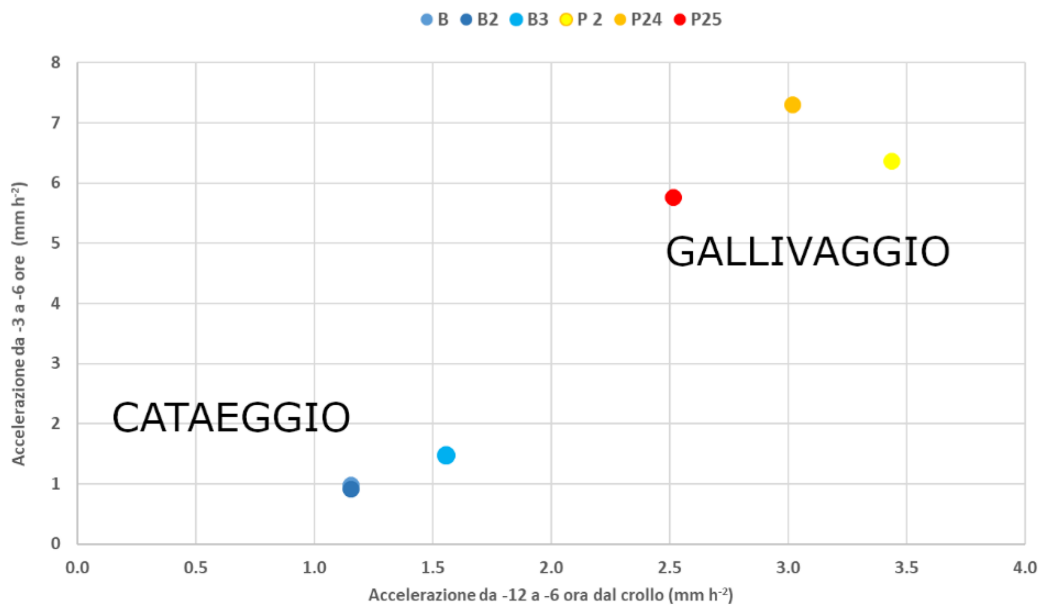

Figure 14. (a) Accelerazione media oraria dell'ultima ora vs accelerazione media oraria a 3 ore dall'evento; (b) Accelerazione media oraria a 3 ore dall'evento vs accelerazione media oraria a 6 ore dall'evento; (c) Accelerazione media oraria a 6 ore dall'evento vs accelerazione media oraria a 12 ore dall' evento. 

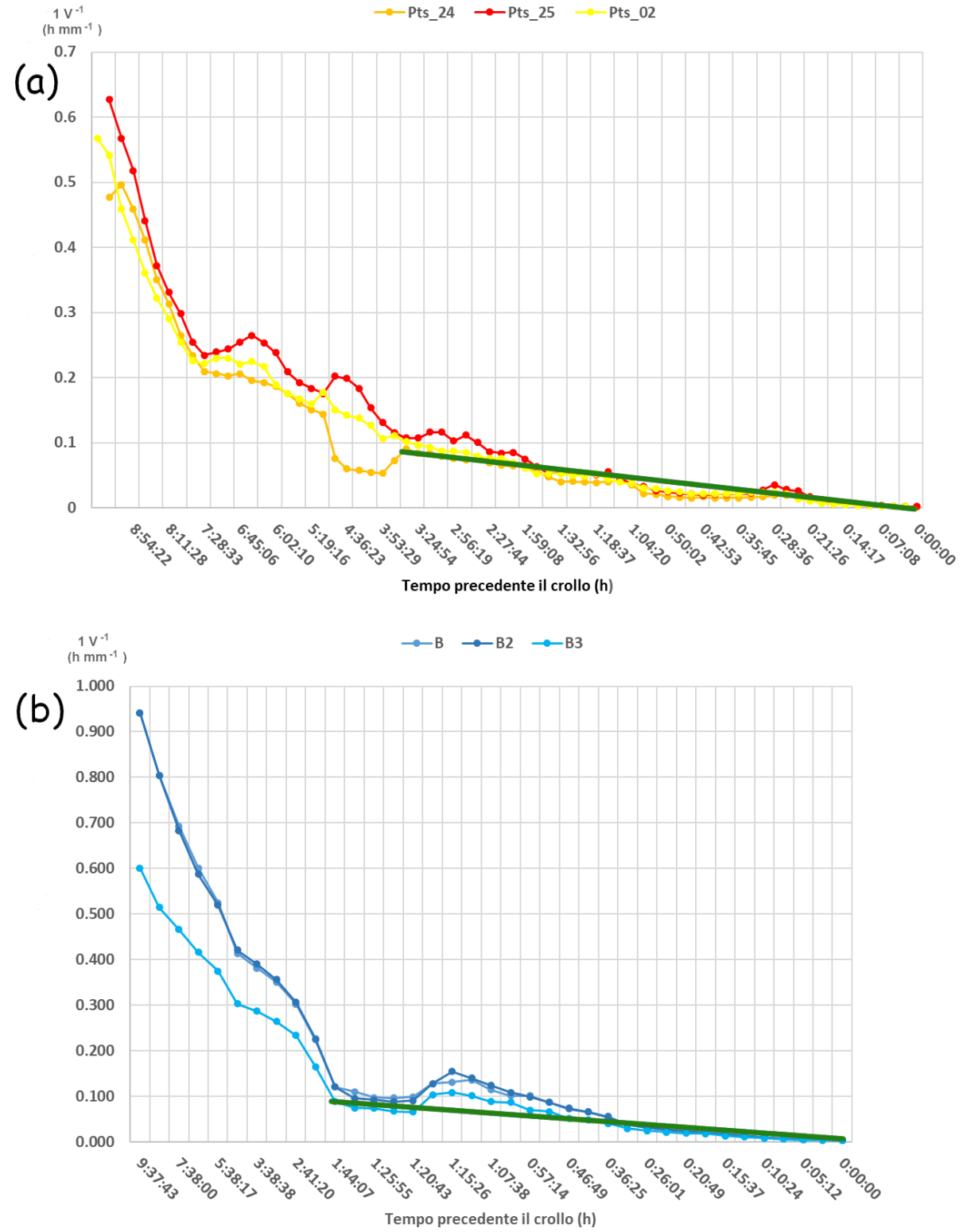

Figure 15. Applicazione del metodo dell'inverso della velocità sui dati di (a) Gallivaggio e (b) Cataeggio, corretti mediante processo di srotolamento della fase, relativi alle velocità istantanee dei singoli punti.

studio. La possibilità che l'interferometria ha di restituire una informazione areale è particolarmente importante, in quanto consente di rilevare situazioni di movimento sull'intera parete e non solo su singoli punti ove siano stati posizionati precedentemente mire ottiche o sensori. Detta informazione areale permette di individuare tutti gli eventi che abbiano caratteristiche volumetriche tali da poter essere analizzati dall'interferometria.

La realizzazione e gestione di una rete non è sufficiente, di per sé, a dispiegare tutti i positivi effetti dell'early warning se non viene accompagnata da una pianificazione preventiva, che permetta di mettere tempestivamente in atto le dovute procedure di protezione civile. A tal proposito il caso di studio di Gallivaggio può essere portato quale virtuoso esempio dove la rete di monitoraggio ha preventivamente evidenziato l'incipiente franamento; le azioni successive messe in atto dal decisore pubblico hanno fatto sì che l'area sottoposta a pericolo fosse evacuata, ne fosse interdetto l'accesso e venissero rimossi e messe al sicuro alcune opere d'arte presenti.

Con riferimento alle soglie d'allertamento (Sättele et al., 2016) l'innesco dei due scivolamenti traslazionali, pur avendo parecchie similitudini, ha evidenziato comportamenti differenti che suggeriscono cautela nella generalizzazione dei dati acquisiti precedentemente al distacco. In particolare l'esame delle curve di spostamento, di velocità media oraria e 
i rapporti fra accelerazioni, hanno evidenziato una tempistica differente fra il franamento di Gallivaggio e quello di Cataeggio. Se nel primo caso la soglia di $3 \mathrm{~mm}$ in $24 \mathrm{~h}$ è stata superata parecchi giorni prima del crollo, a Cataeggio tale velocità è stata raggiunta solo nella giornata precedente il crollo. L'accelerazione per Gallivaggio è stata costante e marcata e l'utilizzo del metodo dell'inverso della velocità ha visto una convergenza dei risultati tra 4 e 6 ore prima del crollo a differenza di Cataeggio dove tale convergenza si è avuta solamente tra $1 \mathrm{~h}$ e $40 \mathrm{~min}$ e $1 \mathrm{~h} \mathrm{e} 15 \mathrm{~min}$. Inoltre si è ottenuto un rapporto elevato e pressochè costante fra le accelerazioni dei due eventi, come già analizzato in precedenti lavori sperimentali su franamenti in miniera (Carlà et al., 2017), solo nelle ultime 3 ore quando il margine di sicurezza per eventuali azioni di protezione civile potrebbe risultare troppo ristretto.

Anche dal confronto fra i succitati eventi si conferma quindi la difficoltà, derivata da una incertezza del modello o più in generale dei meccanismi che governano il distacco in roccia, nel definire soglie di velocità o accelerazione troppo prossime al momento del distacco. La non completa conoscenza a priori del comportamento duttile o fragile dell'ammasso roccioso e delle fratture, unitamente alla persistenza delle stesse e a una maggior o minore complessità dei piani di taglio (Eberhardt, 2008), rendono particolarmente complicato valutare a priori i tempi di sviluppo del creep terziario.

Per tale ragione e in tali contesti vengono comunemente utilizzati valori soglia sufficientemente cautelativi così da poter limitare i potenziali effetti negativi dei fattori di incertezza insiti nella modellazione, anche se tale assunto potrebbe limitare l'efficacia $(E)$ del sistema di monitoraggio in quanto può determinare un maggior valore di PFA.

Va inoltre detto che la nota asimmetria, stabilita dal Codice Penale, fra mancato e procurato allarme, unitamente alle summenzionate incertezze scientifiche, devono necessariamente determinare comportamenti cautelativi proprio perché non si può escludere, su basi scientificamente affidabili, l'avverarsi di fenomeni con caratteristiche maggiormente negative di quanto ipotizzato (Picialli, 2013).

Infine, per una complessiva disamina della gestione del rischio in prossimità di siffatte pareti rocciose, è importante indagare i costi, sostenuti nelle aree oggetto di studio negli ultimi trent'anni, per la realizzazione di opere di difesa. Tali importi possono essere confrontati con quelli necessari per l'attività decennale di un ipotetico sistema di early warning gestito fino all'emanazione di segnalazioni di allerta con valenza di protezione civile. Tale confronto ci permette di affermare che un anno di monitoraggio per l'early warning comporta un aumento, rispetto a quanto speso negli ultimi anni in opere, di un importo pari all' $1.2 \%$ per Gallivaggio e all' $1.3 \%$ per Cataeggio.

Per tutte queste ragioni le esperienze di Gallivaggio e Cataeggio suggeriscono come, per una migliore gestione del rischio, sia opportuno abbinare alle numerose opere di difesa un adeguato monitoraggio geologico. Questa soluzione sarà tanto più valida quanto più si affianchi all'efficacia $(E)$ della rete una completa gestione della stessa e una preventiva pianificazione delle attività di protezione civile.

Quanto sopra detto non azzererà però il rischio, salvo che non si vogliano mettere in atto interventi di dubbia compatibilità sia economica sia sociale, e andrà perciò sempre tenuto conto che le attività e gli utilizzi del territorio dovranno essere pianificati in modo da risultare compatibili alla pericolosità presente.

Data availability. I dati utilizzati sono disponibili negli archivi di ARPA Lombardia che ne consente l'accesso secondo le modalità in vigore presso l'Agenzia. Per l'accesso civico generalizzato a dati ed informazioni la richiesta deve essere presentata, utilizzando l'apposito modulo, all'Ufficio Relazioni con il Pubblico (URP) Sede Centrale ARPA Lombardia. L'interessato deve indicare con chiarezza a quali, dati, informazioni o documenti intenda accedere. La richiesta può essere trasmessa in uno dei seguenti modi:

- Via Posta Elettronica Certificata (PEC) all'indirizzo: arpa@pec.regione.lombardia.it,

- Via posta elettronica ordinaria all'indirizzo: accessocivico@arpalombardia.it*.

Author contributions. L'ideazione el a concettualizzazione degli argomenti è stata a cura di LDC e MLP. L'elaborazione dei dati è stata effettuata da AP, LDC, l'Indagine sul terreno da AP, NP e la successiva fase di validazione da LDC, AP, NP. La scrittura e la preparazione della bozza originale da LDC con il contributo di tutti i coautori Infine scrittura, revisione e modifica finale dei testi da LDC, MLP, NP, AP.

Competing interests. Gli autori dichiarano di non avere conflitti di interessi.

Acknowledgements. Per la documentazione messa a disposizione, le informazioni e la preziosa collaborazione si ringraziano Benedetto Del Simone, Giacomino Rebuzzi, Massimo Ceriani, Carlo Rivolta, Danilo Grossi, Giovanni Songini, Domenico Jobizzi, Adamo Lisignoli, Andrea Tedoldi, Vincenzo Giarratana, e Daniela Dell'Era. Per la preziosa attività svolta prima, durante e dopo gli eventi franosi in oggetto un particolare ringraziamento va ai colleghi del CMG di Sondrio Michele Aili, Ugo Agnelli, Nicoletta Bondio, Dennis Bonetti, Francesco Ferrarini, Francesco Giudes, Bruno Quadrio, a quelli della U. O. CREO Enrico Zini, Dario Bellingeri, ed Alessandro Menin. Infine, per i preziosi suggerimenti, si ringraziano i due anonimi revisori.

Review statement. This paper was edited by Cristian Scapozza and reviewed by two anonymous referees. 


\section{References}

APAT - Agenzia per la Protezione dell'Ambiente e per i servizi Tecnici: Atlante delle opere di sistemazione dei versanti, Manuali e linee guida 10/2002, 1-132, available at: https://www.isprambiente.gov.it/contentfiles/ 00003400/3486-atlante-versanti-2edizione.pdf (last access: November 2020), 2002.

Barbolini, M.: Interventi di messa in sicurezza dell'abitato di Cataeggio dai crolli del versante Scaiùn in Comune di Val Masino, Progetto esecutivo, ottobre 2018, documento di lavoro, 2018.

Cancelli, P., Bragonzi, G., Adamoli, C., e Anselmini, C.: Opere di difesa per la mitigazione del rischio da caduta massi sull'area di Gallivaggio (San Giacomo Filippo, So), Comunità Montana della Valchiavenna, Regione Lombardia, 2018.

Carlà, T., Intrieri, E., Farina, P., and Casagli, N.: A new method to identify impending failure in rock slopes, Int. J. Rock Mech. Min. Sci., 93, 76-81, https://doi.org/10.1016/j.ijrmms.2017.01.015, 2017.

Cruden, D. M. and Varnes, D. J.: Landslide Types and Processes, Transportation Research Board, Special Report 247, US National Academy of Sciences, Special Report, Transportation Research Board, National Research Council, 36-57, 1996.

Dei Cas, L.: Guidelines for designing, managment and maintenance of the geologic monitoring systems, Rend, Online Società Geologica Italiana, 30, 16-18, https://doi.org/10.3301/ROL.2014.04, 2014.

Dei Cas, L., Pastore, M. L., and Rivolta, C.: Gallivaggio landslide: the geological monitoring, of a rock cliff, for early warning system, Ital. J. Geol. Environ., 2, 41-55, https://doi.org/10.4408/IJEGE.2018-02.O-03, 2018.

Del Simone, B.: Realizzazione della pista di accesso e delle opere per la messa in sicurezza del centro abitato di Cataeggio dal crollo dello Scaiùn, Relazione tecnica, luglio 2016, documento di lavoro, 2016a.

Del Simone, B.: Ripristino dei danni causati dalla frana di crollo della bastionata dello Scaiùn del 15.01.2016, Relazione tecnica, febbraio 2016, documento di lavoro, 2016b.

Eberhardt, E.: Twenty-ninth Canadian Geotechnical Colloquium: the role of advanced numerical methods and geotechnical field measurements in understanding complex deep-seated rock slope failure mechanisms, Can. Geotech. J., 45, 484-510, https://doi.org/10.1139/T07-116, 2008.

Erba, M.: Progetto lavori svaso e difese spondali parte alta della Val Materlo a salvaguardia incolumità pubblica degli abitati di Cataeggio e Filorera, giugno 1988, documento di lavoro, 1988.

Federico, A., Popescu, M., Elia, G., Fidelibus, C., Interno, G., and Murianni, A.: Prediction of time to slope failure: a general framework, Environ. Earth Sci., 66, 245-256, https://doi.org/10.1007/s12665-011-1231-5, 2012.

Ferrari, F., Apuani, T., e Giani, G. P.: Applicazione di modelli cinematici per lo studio delle frane di crollo nella media Val San Giacomo, GEAM, 132, 55-63, 2011.

Frattini, P. e Crosta, G. B.: Valutazione dell' accettabilità del rischio da frana e analisi costi-benefici, Giornale di Geologia Applicata 4, 49-56, 2006.
Fukuzono, T.: A new method for predicting the failure time of a slope, in: Proceedings of the 4th International Conference and Field Workshop in Landslides, Tokyo, 145-150, 1985.

Gabrielli, F.: Protezione civile e responsabilità nella società del rischio: chi valuta, chi decide, chi giudica, in: Protezione civile e responsabilità nella società del rischio, Roma, Biblioteca Nazionale Centrale, 4 ottobre 2011, Dipartimento di Protezione Civile e Fondazione CIMA, Edizioni ETS, 3-10, 2013.

Grossi, D.: Relazione sopralluogo in località Cataeggio per verifica situazione dissesto a seguito di frana di crollo dalla bastionata dello Scaiùn, 19 gennaio 2016, documento di lavoro, 2016 a.

Grossi, D.: Relazione sopralluogo in località Cataeggio per verifica situazione dissesto a seguito di nuovo crollo dalla bastionata dello Scaiùn, 11 febbraio 2016, documento di lavoro, 2016b.

Hoek, E. and Bray, J.: Rock Slope Engineering, 3rd Revised Edn., Institution of Mining and Metallurgy, Chapman \& Hall, London, 360 pp., 1981.

Intrieri, E., Gigli, G., Casagli, N., and Nadim, F.: Brief communication "Landslide Early Warning System: toolbox and general concepts", Nat. Hazards Earth Syst. Sci., 13, 85-90, https://doi.org/10.5194/nhess-13-85-2013, 2013.

Klose, M., Highland, L., Damm, B., and Terhorst, B.: Estimation of direct landslide costs in industrialized countries: challenges, concepts, and case study, in: Landslide Science for a Safer Geoenvironment, Vol. 2: Methods of landslide studies, edited by: Sassa, K., Canuti, P., and Yin, Y., Springer, 661-667, https://doi.org/10.1007/978-3-319-05050-8, 2014.

Leroi, E., Bonnard, C., Fell, R., and McInnes, R.: State of the art: Risk Assessment and Managment, in: Landslide Risk management, edited by: Hungr, O., Fell, R., Countre, R., and Eberhart, E., Balkema, Rotterdam, 159-198, 2005.

Macciotta, R., Martin, C. D., Morgenstern, N. R., and Cruden, D. M.: Quantitative risk assessment of slope hazards along a section of railway in the Canadian Cordillera - a methodology considering the uncertainty in the results, Landslides, 13, 115-127, https://doi.org/10.1007/s10346-014-0551-4, 2016.

Menegoni, N., Giordan, D., and Perotti, C. : Reliability and Uncertainties of the Analysis of an Unstable Rock Slope Performed on RPAS Digital Outcrop Models: the Case of the Gallivaggio Landslide (Western Alps, Italy), Remote Sens., 12, 1-25, https://doi.org/10.3390/rs12101635, 2020.

Picialli, P.: La protezione civile nella società del rischio: procedure, garanzie, responsabilità, in: La Protezione civile nella società del rischio - Procedure, garanzie, responsabilità, Milano, Palazzo di Giustizia, 30 novembre 2013, Dipartimento di Protezione Civile e Fondazione CIMA, Edizioni ETS, 57-68, 2013.

Sättele, M., Bründl, M., and Straub, D.: Reliability and effectiveness of early warning systems for natural hazards: Concept and application to debris flow warning, Reliab. Eng. Syst. Safe., 142, 192-202, https://doi.org/10.1016/j.ress.2015.05.003, 2015.

Sättele, M., Krautblatter, M., Bründl, M., and Straub, D.: Forecasting rock slope failure: how reliable and effective are warning systems?, Landslides, 13, 737-750, https://doi.org/10.1007/s10346015-0605-2, 2016.

Scaramellini, G.: La Madonna di Gallivaggio, Storia e arte, Edizione Gallivaggio, 159 pp., 1998.

Schmid, S. M., Berger A., and Davidson C.: The Bergell pluton (Southern Switzerland, Northern Italy): overview accompanying a geological-tectonic map of the intrusion and surrounding coun- 
try rocks, Schweizerische mineralogische und petrographische Mitteilungen, 76, 329-355, 1996.

Songini, G.: Manutenzione straordinaria delle opere idrauliche esistenti e realizzazione di un vallo paramassi a monte degli abitati di Cataeggio e Filorera nella Val Materlo, Relazione Geologica, luglio 2010, documento di lavoro, 2010.

Songini, G.: Manutenzione straordinaria delle opere idrauliche esistenti e realizzazione di un vallo paramassi a monte degli abitati di Cataeggio e Filorera nella Val Materlo-Variante in corso d'opera e suppletive, Relazione Geologica, settembre 2011, documento di lavoro, 2011.

Songini, G.: Scheda CLPV Val Masino numero 7140740056, luglio 2000, documento di lavoro, 2000.
Varnes, D.J.: Slope Movements Types and Processes, in: Landslides Analiysis and Control Trasportation Research Board, Special Report 176, chap. 2, National Academy of Sciences, Washington, D.C., 11-33, 1978.

Varnes, D. J.: Commission on landslide and other mass movements IAEG - Landslide hazard zonation a review of principles and practice, UNESCO Press, Paris, 63 pp., 1984.

Voight, B.: A relation to describe rate-dependent material failure, Science, 243, 200-203, https://doi.org/10.1126/science.243.4888.200, 1989. 